\title{
Fiber-Based Optical Parametric Amplifiers and Their Applications
}

\author{
Jonas Hansryd, Peter A. Andrekson, Member, IEEE, Mathias Westlund, Jie Li, and Per-Olof Hedekvist
}

Invited Paper

\begin{abstract}
An applications-oriented review of optical parametric amplifiers in fiber communications is presented. The emphasis is on parametric amplifiers in general and single pumped parametric amplifiers in particular. While a theoretical framework based on highly efficient four-photon mixing is provided, the focus is on the intriguing applications enabled by the parametric gain, such as all-optical signal sampling, time-demultiplexing, pulse generation, and wavelength conversion. As these amplifiers offer high gain and low noise at arbitrary wavelengths with proper fiber design and pump wavelength allocation, they are also candidate enablers to increase overall wavelength-division-multiplexing system capacities similar to the more well-known Raman amplifiers. Similarities and distinctions between Raman and parametric amplifiers will also be addressed. Since the first fiber-based parametric amplifier experiments providing net continuous-wave gain in the for the optical fiber communication applications interesting $1.5-\mu \mathrm{m}$ region were only conducted about two years ago, there is reason to believe that substantial progress may be made in the future, perhaps involving "holey fibers" to further enhance the nonlinearity and thus the gain. This together with the emergence of practical and inexpensive high-power pump lasers may in many cases prove fiber-based parametric amplifiers to be a desired implementation in optical communication systems.
\end{abstract}

Index Terms-Nonlinear optics, fiber-optic amplifiers and oscillators, O-TDM, multiplexing, demultiplexing, optical sampling.

\section{INTRODUCTION}

$\mathbf{P}$ ARAMETRIC amplification is a well-known phenomenon in materials providing $\chi^{(2)}$ nonlinearity [1]. However, parametric amplification can also be obtained in optical fibers exploiting the $\chi^{(3)}$ nonlinearity. New high-power light sources and optical fibers with a nonlinear parameter 5-10 times higher than for conventional fibers [2], [3], as well as the need of am-

Manuscript received February 12, 2002; revised March 27, 2002. This work was supported in part by the Swedish Strategic Research Foundation (SSF), the Swedish Research Council (VR), and Chalmers Center for High-Speed Technology $(\mathrm{CHACH})$.

J. Hansryd was with Chalmers University of Technology, Photonics Laboratory, Department of Microelectronics MC2, SE-412 96 Göteborg, Sweden. He is now with CENiX Inc., Allentown, PA USA 18106 (e-mail: jhansryd@cenix.com).

P. A. Andrekson is with Chalmers University of Technology, SE-41296 Göteborg, Sweden and also with CENiX Inc., Allentown, PA 18106 USA.

M. Westlund and P.-O. Hedekvist are with Chalmers University of Technology, SE-412 96 Göteborg, Sweden.

J. Li is with Chalmers University of Technology, SE-412 96 Göteborg,

Sweden and also with Ericsson Telecom AB, Stockholm, Sweden.

Publisher Item Identifier S 1077-260X(02)05481-3. plification outside the conventional Erbium band has increased the interest in such optical parametric amplifiers (OPA). The fiber-based OPA is a well-known technique offering discrete or "lumped" gain using only a few hundred meters of fiber [4], [5]. It offers a wide gain bandwidth and may in similarity with the Raman amplifier [6] be tailored to operate at any wavelength [7]-[11]. Although continuous-wave (CW) pumped fiber OPAs have been experimentally investigated since the late 1980s [12], it was not until about two years ago that net "black-box gain" was achieved in the $1.5-\mu \mathrm{m}$ region. An OPA is pumped with one or several intense pump waves providing gain over two wavelength bands surrounding the single pump wave, or in the latter case, the wavelength bands surrounding each of the pumps. As the parametric gain process do not rely on energy transitions between energy states it enable a wideband and flat gain profile contrary to the Raman and the Erbium-doped fiber amplifier (EDFA). The underlying process is based on highly efficient four-photon mixing (FPM) ${ }^{1}$ relying on the relative phase between four interacting photons [13]-[16]. Due to the phase matching condition, the OPA does not only offer phase-insensitive amplification, but also the important feature of phase-sensitive parametric amplification. The phase-sensitive amplifier only amplifies components of the same phase as the signal, while attenuating components with the opposite phase [9], [17], [18]. This property has many potential uses, e.g., pulse reshaping [19], [20], as well as dispersive wave, soliton-soliton interaction, and quantum noise suppression [21]-[23]. Another very important application is the possibility of in-line amplification with an ideal noise figure of $0 \mathrm{~dB}$ [18], [24], [25]. This should be compared to the quantum limited noise figure of $3 \mathrm{~dB}$ for standard phase-insensitive amplifiers. A difficult but necessary requirement for phase-sensitive OPAs is the need for a strict control of the phases of all involved light waves. The most usual experimental implementation of such an amplifier is thus through a nonlinear Kerr interferometer where the phase of only one light source needs to be tracked [17], [26].

For the phase-insensitive OPA, two photons at one or two pump wavelengths with arbitrary phases will interact with a signal photon. A fourth photon, the idler, will be formed with a phase such that the phase difference between the pump photons and the signal and idler photon satisfies a phase matching condition (this is further discussed below). The phase-insensitive

\footnotetext{
${ }^{1}$ In the literature, four-photon mixing is also referred to as four-wave mixing.
} 


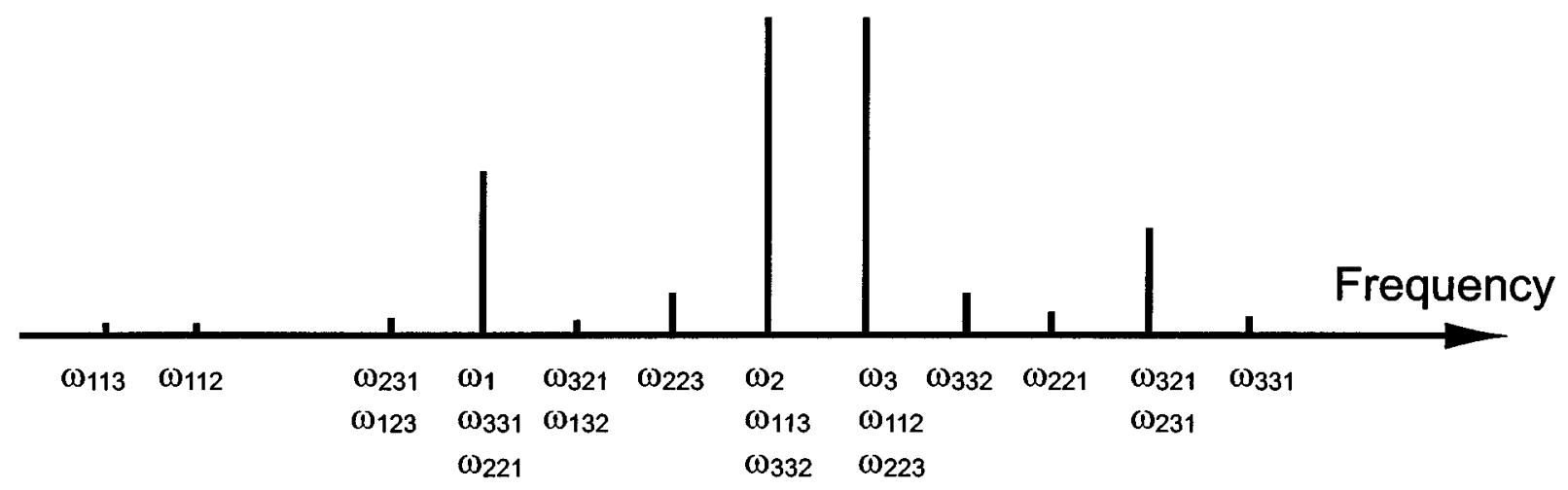

Fig. 1. Frequency components generated due to FPM for two pumps at frequencies $\omega_{2}$ and $\omega_{3}$ and a weak signal at $\omega_{1}$.

OPA lacks the ability of amplification with a subquantum-limited noise figure, while the requirements for its implementation are substantially relaxed and it still offers the important properties of high differential gain, optional wavelength conversion and operation at an arbitrary wavelength.

As the Kerr effect, similarly to the Raman process, relies on nonlinear interactions in the fiber, the intrinsic gain response time for an OPA is in the same order as for the Raman amplifier (a few femtoseconds). This prevents in many, but not all cases, the amplifier from operating in a saturated mode. In return, it allows for ultrafast all-optical signal processing. Potential applications, further discussed below, include in-line amplification [4], [5], return-to-zero (RZ)-pulse generation [27], optical time-division demultiplexing (O-TDM) [28], [29], transparent wavelength conversion [10], [11], all-optical limiters [28], [30], and all-optical sampling [31].

The remaining part of this paper describes the theory and the possible applications of phase-insensitive fiber based parametric amplifiers to high-speed and long-haul transmission systems. The focus is on degenerated parametric amplification using one strong pump at a single wavelength, while the results in general are possible to extend to two pumps at different frequencies [8], [13], [32], [33], [53].

In Section II, we will describe the theory, the limitations and advantages of fiber based OPAs, and also give a brief comparison with the well known Raman amplifier. In Section III, we will discuss and demonstrate some general applications of the OPA. We will start with a CW pumped linear amplifier and a transparent wavelength converter followed by a review of experiments demonstrating high speed O-TDM applications. The paper is concluded with a brief discussion on future developments.

\section{THEORY}

\section{A. Four-Photon Mixing}

To understand the parametric gain process, relying on highly efficient FPM, we will describe it from three view angles. In an intuitive approach, the nondegenerated process starts with two waves at frequencies $\omega_{1}$ and $\omega_{2}$ that copropagate together through the fiber. As they propagate they will continuously beat with each other. The intensity modulated beat note at frequency $\omega_{2}-\omega_{1}$ will modulate the intensity dependent refractive index $n(\omega, I)$ of the fiber. When a third wave at frequency $\omega_{3}$ is added, it will become phase modulated (PM) with the frequency $\omega_{2}-$ $\omega_{1}$, due to the modulated $n$. From the PM, the wave at $\omega_{3}$ will develop sidebands at the frequencies $\omega_{3} \pm\left(\omega_{2}-\omega_{1}\right)$. The amplitude of the sidebands will be proportional to the amplitude of the signal at $\omega_{3}$. In the same way, $\omega_{3}$ will beat with $\omega_{1}$ and PM $\omega_{2}$. As a consequence the wave at $\omega_{2}$ will generate sidebands at $\omega_{2} \pm\left(\omega_{3}-\omega_{1}\right)$, where $\omega_{2}+\left(\omega_{3}-\omega_{1}\right)$ will coincide with the previously mentioned $\omega_{3}+\left(\omega_{2}-\omega_{1}\right)$. It should be noted that from a FPM process including three incident waves and all possible degenerated and partially degenerated processes, nine new frequencies will be generated [34]. Fig. 1 shows all nine frequencies. It also shows that some FPM-products will overlap with the signal frequency, in this case $\omega_{1}$. These products will result in a gain for the signal, i.e., provide parametric amplification. In general, the remaining weaker frequencies are usually neglected with the exception of the stronger frequency component at $\omega_{4}=\omega_{3}+\left(\omega_{2}-\omega_{1}\right)=\omega_{2}+\left(\omega_{3}-\omega_{1}\right)$. The two overlapping components $\left(\omega_{321}\right.$ and $\left.\omega_{231}\right)$ at $\omega_{4}$ are here referred to as the generated idler. In the degenerated case with one pump, $\omega_{2}$ and $\omega_{3}$ will coincide and light will only be transferred to the signal and the idler frequency.

For the rest of this chapter, we will focus on the degenerated case including one pump at $\omega_{p}$, one signal at $\omega_{s}$ and one idler lightwave at $\omega_{i}$. From the above discussion it follows that a requirement for the FPM process to be "resonant" is that both a phase-matching condition between the waves is maintained, and that the frequencies of the three waves are symmetrically positioned relatively to each other,

$$
\begin{aligned}
\Delta \beta & =\beta\left(\omega_{s}\right)+\beta\left(\omega_{i}\right)-2 \beta\left(\omega_{p}\right)=0 \\
2 \omega_{p} & =\omega_{s}+\omega_{i} .
\end{aligned}
$$

Here, $\Delta \beta$ is the low power propagation mismatch, $c$ is the speed of light in vacuum and $\beta_{s, i, p}=\omega_{s, i, p} n\left(\omega_{s, i, p}\right) / c$ is the propagation constant of each lightwave.

Parametric amplification can be viewed from a quantum mechanical picture. Here, the degenerated parametric amplification is manifested as the conversion of two pump photons at frequency $\omega_{p}$ to a signal and an idler photon at frequencies $\omega_{s}$ and $\omega_{i}$. The conversion needs to satisfy the energy conservation relation as in (2) and the quantum-mechanical photon momentum conservation relation as in (1).

From an electromagnetic point of view we may consider the interaction of three stationary copolarized waves at an- 
gular frequencies $\omega_{p}, \omega_{s}$ and $\omega_{i}$, characterized by the slowly varying electric fields with complex amplitudes $A_{p}(z), A_{s}(z)$ and $A_{i}(z)$, respectively. The total transverse field $E(x, y, z)$ propagating along the single-mode fiber may be written as [16]

$$
\begin{aligned}
E(x, y, z)= & f(x, y) A(z)=f(x, y) \frac{1}{2}\left[A_{p}(z)\right. \\
& \times \exp \left(i \beta_{0} z-i \omega_{0} t\right)+A_{s}(z) \exp \left(i \beta_{1} z-i \omega_{1} t\right) \\
& \left.+A_{i}(z) \exp \left(i \beta_{2} z-i \omega_{2} t\right)+c . c\right]
\end{aligned}
$$

where c.c is the complex conjugate which is usually omitted in the calculations and $f(x, y)$ is the common transverse modal profile which is assumed to be identical for all three waves along the fiber. Using the basic propagation equation [35], it is straightforward to derive three coupled equations for the complex field amplitude of the three waves $A_{p, s, i}$ [13], [14], [16], [36]

$$
\begin{aligned}
\frac{d A_{p}}{d z}= & i \gamma\left[\left(\left|A_{p}\right|^{2}+2\left(\left|A_{s}\right|^{2}+\left|A_{i}\right|^{2}\right)\right) A_{p}\right. \\
& \left.+2 A_{s} A_{i} A_{p}^{*} \exp (i \Delta \beta z)\right], \\
\frac{d A_{s}}{d z}= & i \gamma\left[\left(\left|A_{s}\right|^{2}+2\left(\left|A_{i}\right|^{2}+\left|A_{p}\right|^{2}\right)\right) A_{s}\right. \\
& \left.+A_{i}^{*} A_{p}^{2} \exp (-i \Delta \beta z)\right], \\
\frac{d A_{i}}{d z}= & i \gamma\left[\left(\left|A_{i}\right|^{2}+2\left(\left|A_{s}\right|^{2}+\left|A_{p}\right|^{2}\right)\right) A_{i}\right. \\
& \left.+A_{s}^{*} A_{p}^{2} \exp (-i \Delta \beta z)\right] .
\end{aligned}
$$

Here, fiber loss have been neglected and $\gamma=2 \pi n_{2} / \lambda A_{\text {eff }}$ is the nonlinearity coefficient where $n_{2}$ is the fiber nonlinear parameter and $A_{\text {eff }}$ is the effective modal area of the fiber. Furthermore, the frequencies are assumed to be similar such that $\gamma$ are equal for the three light waves and $n_{2}$ is assumed approximately real such that any Raman gain is negligible. The first two terms on the right-hand side (RHS) of (4)-(6) are responsible for the nonlinear phase shift due to self-phase modulation (SPM) and cross-phase modulation (XPM), respectively. The last term is responsible for the energy transfer between the interacting waves. If required, fiber loss may be included by adding the loss term $-\left.(\alpha / 2) A_{j}\right|_{j=p, s, i}$ to the RHS of each equation, respectively.

\section{B. Phase-Sensitive or Phase-Insensitive Parametric Amplification}

By rewriting (4)-(6) in terms of powers and phases of the waves further insight can be gained. Let $P_{p, s, i}(z)=\left|A_{p, s, i}(z)\right|^{2}$ and $\phi_{p, s, i}(z)$, where $A_{j}(z)=\sqrt{P_{j}} \exp \left(i \phi_{j}\right)$ for $j \in\{p, s, i\}$ [14], [36], [37].

$$
\begin{aligned}
\frac{d P_{p}}{d z}= & -4 \gamma\left(P_{p}^{2} P_{s} P_{i}\right)^{1 / 2} \sin \theta \\
\frac{d P_{s}}{d z}= & 2 \gamma\left(P_{p}^{2} P_{s} P_{i}\right)^{1 / 2} \sin \theta \\
\frac{d P_{i}}{d z}= & 2 \gamma\left(P_{p}^{2} P_{s} P_{i}\right)^{1 / 2} \sin \theta \\
\frac{d \theta}{d z}= & \Delta \beta+\gamma\left(2 P_{p}-P_{s}-P_{i}\right)+\gamma\left[\left(P_{p}^{2} P_{i} / P_{s}\right)^{1 / 2}\right. \\
& \left.+\left(P_{p}^{2} P_{i} / P_{s}\right)^{1 / 2}-4\left(P_{s} P_{i}\right)^{1 / 2}\right] \cos \theta .
\end{aligned}
$$

Here, $\theta(z)$ describes the relative phase difference between the four involved light waves

$$
\theta(z)=\Delta \beta z+2 \phi_{p}(z)-\phi_{s}(z)-\phi_{i}(z)
$$

where $\phi_{j}(z)$ includes both the initial phase at $z=0$ and the acquired nonlinear phase shift during propagation.

The first term of $\theta$ on the RHS of (10) describes the linear phase shift, while the second and third term describe the nonlinear phase shift. The attentive reader may note that a phase term representing the time dependent phase difference, $\Delta \phi(t)$ is missing in (11). However, this term $\Delta \phi(t)=2 \omega_{p} t-\omega_{s} t-$ $\omega_{i} t$ will remain zero when $\omega_{s}+\omega_{i}=2 \omega_{p}$, resulting in the phase-matching condition previously stated in (2).

As can be observed from (7)-(10), by controlling the phase relation $\theta$, we have the opportunity to control the direction of the power flow from the pump to the signal and the idler $(\theta=\pi / 2$, parametric amplification) or from the signal and the idler to the pump $(\theta=-\pi / 2$, parametric attenuation). In other words, by having signal, idler, and pump photons present at the fiber input and adjusting the relative phase between them, we are able to decide if the signal will be amplified or attenuated. This gives us the possibility to create a phase-sensitive amplifier. As previously discussed in the introductory part of this paper, the major obstacle for implementing such device is the difficulties of controlling and maintaining the relative phase of the interacting photons.

For the general application of a phase-insensitive fiber-based parametric amplifier as outlined in Fig. 2, we may consider an intense pump at $\omega_{p}$ and a weak signal at $\omega_{s}$. The idler is assumed to be zero at $z=0$. For this special case $\theta=\pi / 2$ at the fiber input port. This can be understood as described by Inoue and Mukai [37] by realizing that the idler will be generated after an infinitesimal propagation distance in the fiber. Analyzing the phases in (6): $d A_{i}=i \gamma A_{p}^{2} A_{s}^{*} \exp (-i \Delta \beta z) d z$, shows that the phase of the initiated idler will be $\phi_{i}=\pi / 2+2 \phi_{p}-\phi_{s}-$ $\Delta \beta z$; thus $\theta=\pi / 2$ at the input port. Following (8)-(9), this has the consequence that the signal and the idler will start to grow immediately in the fiber.

\section{Phase Matching Condition}

Operating in a phase-matched condition $\theta(z)$ remains near $\pi / 2$, the third term in (10) may be neglected and the following approximation, first introduced by Stolen and Bjorkholm in [13] is valid

$$
\frac{d \theta}{d z} \approx \Delta \beta+\gamma\left(2 P_{p}-P_{s}-P_{i}\right) \approx \Delta \beta+2 \gamma P_{p}=\kappa .
$$

Here, the phase mismatch parameter $\kappa$ is introduced and the second approximation is valid when the amplifier is operating in an undepleted mode $\left(P_{p} \gg P_{s}\right)$.

Expanding $\beta(\omega)$ in Taylor series to the fourth order around the zero-dispersion frequency $\omega_{0},\left(\beta_{2}\left(\omega_{0}\right)=0\right)$ the wavelength dependent part, $\Delta \beta$ of the phase mismatch parameter $\kappa$ can be rewritten as

$$
\begin{aligned}
\Delta \beta=\left\{\beta_{3}\left(\omega_{p}-\omega_{0}\right)+\right. & \frac{\beta_{4}}{2}\left[\left(\omega_{p}-\omega_{0}\right)^{2}\right. \\
& \left.\left.+\frac{1}{6}\left(\omega_{p}-\omega_{s}\right)^{2}\right]\right\}\left(\omega_{p}-\omega_{s}\right)^{2} .
\end{aligned}
$$




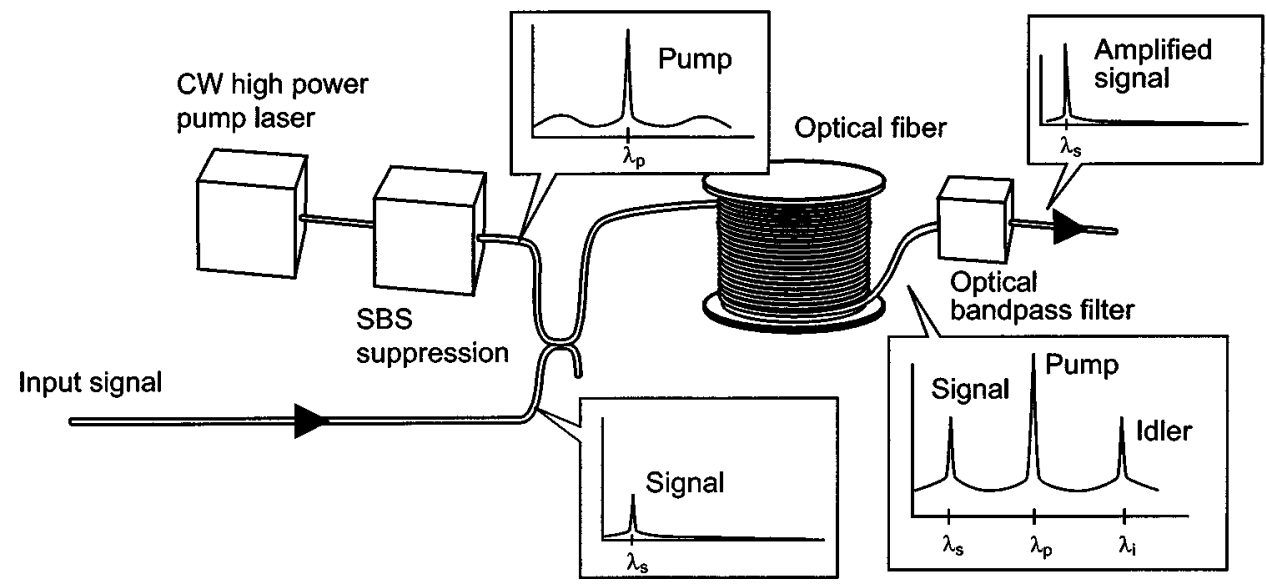

Fig. 2. General scheme of phase-insensitive fiber-based optical parametric amplifier.

Here, $\beta_{3}$ and $\beta_{4}$ is the third and fourth derivative of the propagation constant $\beta(\omega)$ at $\omega_{0}$. When the pump frequency is chosen to $\omega_{p}=\omega_{0}$ the wavelength dependent part, $\Delta \beta$ of the phase mismatch parameter $\kappa$, and thus the OPA gain bandwidth may be limited by the fourth-order dispersion [7]. As $\beta_{4}$ is typically in the $\approx 10^{-4} \mathrm{ps}^{4} / \mathrm{km}$ range, higher order dispersion becomes an important and fundamental limiting factor as the operating bandwidth $\left(\lambda_{p}-\lambda_{s}\right)$ exceeds $100 \mathrm{~nm}$.

By neglecting $\beta_{4}$, a convenient approximative transformation of (13) may be done from the frequency domain to the more generally used wavelength domain [28],

$$
\begin{aligned}
\Delta \beta=\beta\left(\omega_{s}\right)+\beta\left(\omega_{i}\right) & -2 \beta\left(\omega_{p}\right) \\
= & -\frac{2 \pi c}{\lambda_{0}^{2}} \frac{d D}{d \lambda}\left(\lambda_{p}-\lambda_{0}\right)\left(\lambda_{p}-\lambda_{s}\right)^{2} .
\end{aligned}
$$

Here, $d D / d \lambda$ is the slope of the dispersion at the zero-dispersion wavelength and the approximation $\omega_{i}-\omega_{j}=c / 2 \pi \lambda_{0}^{2}\left(\lambda_{j}-\lambda_{i}\right)$ has been made. This approximation is only valid for bandwidths where $\lambda_{p}-\lambda_{s} \ll \lambda_{p}$.

When $\lambda_{p}$ is positioned in the normal dispersion regime $\left(\lambda_{p}<\right.$ $\left.\lambda_{0}\right)$, the accumulated phase mismatch will increase with increasing signal wavelength $\lambda_{s}$, thus decreasing the resulting efficiency of the process. By positioning the pump wavelength in the anomalous dispersion regime $(\Delta \beta<0)$, it is possible to compensate for the nonlinear phase mismatch $2 \gamma P_{p}$ by the linear phase mismatch $\Delta \beta$. For a fixed $\lambda_{p}$, the gain versus signal wavelength $\lambda_{s}$ will thus be formed in two lobes on each side of $\lambda_{p}$, each lobe having its peak gain for $\kappa=\Delta \beta+2 \gamma P_{p}=0$. This process is identical to the phenomenon that is also referred to as modulation instability [32], i.e., the parametric process establishes a balance between GVD and the nonlinear Kerr-effect.

As a sidenote, it can be mentioned that parametric amplification with the pump positioned in the normal dispersion regime may be achieved by using a birefringent fiber [16]. The linear phase mismatch consist of material phase mismatch (due to the properties of fused silica) and waveguide phase mismatch (due to the design of the optical fiber). In the normal dispersion regime, the nonlinear and the material phase mismatch contribution will have the same sign. By placing the pump in the slow propagation axis of the fiber, while the idler and signal is positioned in the fast axis, the sign of the waveguide mismatch contribution will cancel the material and nonlinear mismatch contributions and parametric amplification may occur.

Combining the expression for the maximum power flow i.e., $\kappa=0$ with (14) shows that

$$
2 \gamma P_{p}=-\Delta \beta \propto\left(\lambda_{p}-\lambda_{0}\right)\left(\lambda_{p}-\lambda_{s}\right)^{2} .
$$

Hence, the separation between the gain peaks for the signal wavelength will increase with increasing $P_{p}$ and with $\lambda_{p}$ fixed.

Considering the special case when the pump becomes depleted such that the condition $P_{p} \gg P_{s}$ is no longer fulfilled. Studying (12), we may note that the approximation performed in the last equality is no longer correct. If we still assume that we are operating in the phase matched regime $\theta(z) \sim \pi / 2$, the nonlinear phase mismatch will decrease in order to keep the total phase mismatch close to zero so that the optimum linear phase mismatch will decrease compared to $\Delta \beta$ predicted by (15) with $\kappa=0$. When the pump become so depleted that $\theta(z) \neq \pi / 2$, the power will start to oscillate between the pump and the signal/idler as a consequence of $\theta(z)$ will start to oscillate between $-\pi / 2$ and $\pi / 2$.

Equations (4)-(6) are general in the sense that they include a depleted pump, higher order dispersion and a nonlinear phase shift, they may also easily be solved numerically by using a standard computer math package. An improved understanding can be obtained by considering a strong pump and a weak signal incident at the fiber input such that the pump remains undepleted during the parametric gain process. We may then set $d A_{0} / d z=$ 0 and an analytical solution may be derived for the remaining coupled equations as [13]

$$
\begin{aligned}
& P_{s}(L)=P_{s}(0)\left(1+\left[\frac{\gamma P_{p}}{g} \sinh (g L)\right]^{2}\right) \\
& P_{i}(L)=P_{s}(0)\left[\frac{\gamma P_{p}}{g} \sinh (g L)\right]^{2} .
\end{aligned}
$$

Here, $L$ is the fiber interaction length and the parametric gain coefficient $g$ is given by

$$
g^{2}=\left[\left(\gamma P_{p}\right)^{2}-(\kappa / 2)^{2}\right]=-\Delta \beta\left[\frac{\Delta \beta}{4}+\gamma P_{p}\right] .
$$


If the fiber is long or the attenuation is high, the interaction length will be limited by the effective fiber length $L_{\text {eff }}$ expressed as [38]

$$
L_{\mathrm{eff}}=\frac{1-\exp (-\alpha L)}{\alpha}
$$

where $\alpha$ is the loss coefficient of the fiber. In most applications involving low-loss HNLF $\alpha L \ll 1$ such that $L_{\text {eff }} \approx L$. In the remaining part of this paper that condition will be assumed. The unsaturated single pass gain $G_{s}$ and the unsaturated wavelength conversion efficiency $G_{i}$ may be written [7], [33]

$$
\begin{aligned}
G_{s} & =\frac{P_{s}(L)}{P_{s}(0)}=1+\left[\frac{\gamma P_{p}}{g} \sinh (g L)\right]^{2} \\
& =1+\left(\gamma P_{p} L\right)^{2}\left[1+\frac{g L^{2}}{6}+\frac{g L^{4}}{120}+\cdots\right]^{2} \\
G_{i} & =G_{s}-1 .
\end{aligned}
$$

In (20), the last equality stems from the Taylor expansion of $\sinh (x)$.

From (20), it may be noted that for signal wavelengths close to $\lambda_{p}, \Delta \beta \approx 0$ and $G_{s} \approx\left(\gamma P_{p} L\right)^{2}$. In the special case of perfect phase matching $(\kappa=0)$ and $\gamma P_{p} L \gg 1$, (20) may be rewritten as

$$
\begin{aligned}
G_{s} & \simeq \sinh ^{2}(g L) \simeq \sinh ^{2}\left(\gamma P_{p} L\right) \\
& =\frac{\left[\exp \left(\gamma P_{p} L\right)-\exp \left(-\gamma P_{p} L\right)\right]^{2}}{4} \\
& \simeq \frac{1}{4} \exp \left(2 \gamma P_{p} L\right) .
\end{aligned}
$$

\section{Discussion on Amplifier Gain and Bandwidth}

The above expression shows that for the perfect phase-matching case, the parametric gain is approximately exponentially proportional to the applied pump power. A very simple expression for the OPA peak gain may be obtained if (22) is rewritten in decibel units as

$$
\begin{aligned}
G_{\mathrm{dB}} & =10 \log _{10}\left[\frac{1}{4} \exp \left(2 \gamma P_{p} L\right)\right] \\
& =P_{p} L S_{P}-6
\end{aligned}
$$

where $S_{P}=10 \log _{10}[\exp (2)] \gamma \cong 8.7 \gamma$ is introduced as the parametric gain slope in $[\mathrm{dB} / \mathrm{W} / \mathrm{km}]$. Fig. 3 shows calculated gain for a parametric amplifier with 1.4-W pump power and 500-m HNLF with $\gamma=11 \mathrm{~W}^{-1} \cdot \mathrm{km}^{-1}$. The region for perfect phase matching (exponential parametric gain) and the region where $\Delta \beta \approx 0$ (quadratic parametric gain) is marked in the figure. Fig. 4 shows the measured gain slope $S_{p}$ for the same experimental fiber parameters [5]. The amplifier bandwidth may be defined as the width of each gain lobe surrounding $\lambda_{p}$ [7], [8], [13]. From (14), (18), (20) it may be observed that the amplifier bandwidth for a fixed $\gamma P_{p} L$ will increase with decreasing $L$ as the reduction in $G_{s}$ with respect to $\lambda$ will be "accelerated" by the longer fiber length. On the other hand, since $\gamma P_{p}$ increases as $L$ decreases, the peak gain wavelength will be pushed further away from $\lambda_{p}$. This is an important observation since the available

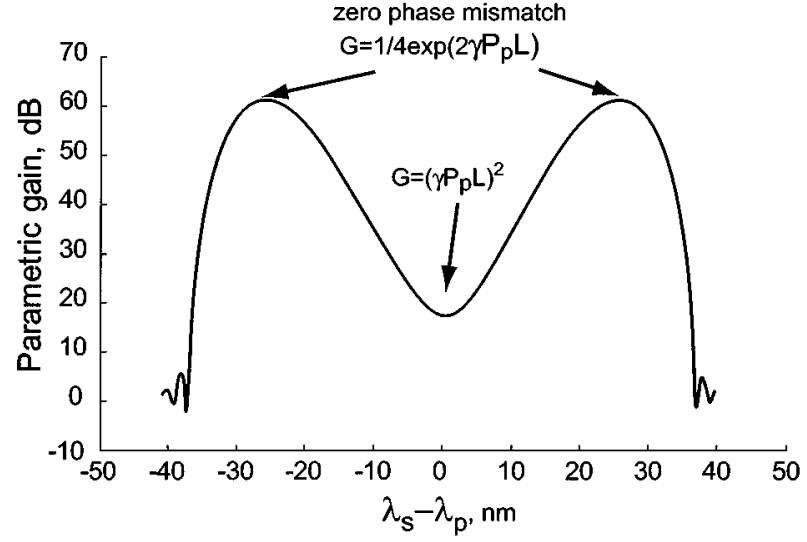

Fig. 3. Calculated gain for a fiber optical parametric amplifier with $P_{p}: 1.4 \mathrm{~W}$, $L: 500 \mathrm{~m}, \gamma: 11 \mathrm{~W}^{-1} \cdot \mathrm{km}^{-1}, \lambda_{0}: 1559 \mathrm{~nm}, \lambda_{p}: 1560.7 \mathrm{~nm}, d D / d \lambda=0.03$ $\mathrm{ps} / \mathrm{nm}^{2} \mathrm{~km}$. Arrows indicate regions with exponential gain and the region with quadratic gain proportional to the applied pump power.

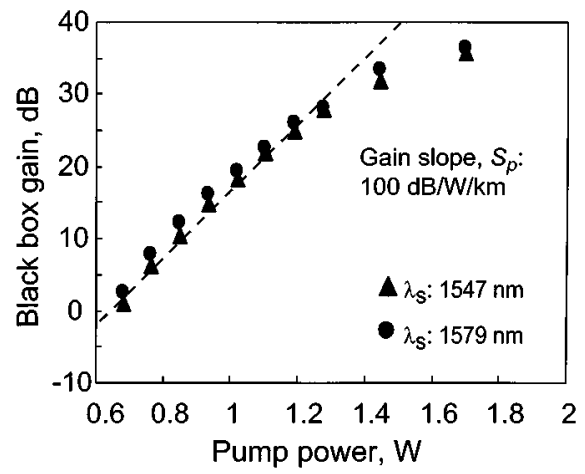

Fig. 4. Measured parametric gain slope $S_{p}$ using 500-m HNLF with $\gamma=$ $11 \mathrm{~W}^{-1} \cdot \mathrm{km}^{-1}, \lambda_{0}=1561.5 \mathrm{~nm}$, and $d D / d \lambda=0.03 \mathrm{ps} / \mathrm{nm}^{2} \mathrm{~km}$. The gain slopes were measured for the two peak wavelengths 1547 and $1579 \mathrm{~nm}$, respectively.

power was usually the limiting factor for conventional optical fibers. By using a short HNLF it is possible to decrease $L$ and increase $\gamma$ such that the maximum gain is fixed while the amplifier bandwidth is increased. The benefit of using such a fiber is demonstrated in Fig. 5. Here, the single pass gain is calculated from (16) for different fiber lengths. The product $\gamma P_{p} L$ is constant, resulting in a fixed maximum gain but an increased bandwidth as the fiber length is decreased. The condition $\gamma P_{p} L=10$ correspond e.g., to a pump power of $1 \mathrm{~W}$ for a HNLF with $L=1000 \mathrm{~m}$ and $\gamma=10 \mathrm{~W}^{-1} \cdot \mathrm{km}^{-1}$. Decreasing the fiber length to $50 \mathrm{~m}(L=0.05 L)$ would increase the bandwidth 20 times and require for instance $P_{p}=4 \mathrm{~W}$ and $\gamma=50$ $\mathrm{W}^{-1} \cdot \mathrm{km}^{-1}$. Such a high $\gamma$ could be achieved in novel types of HNLF such as air-silica microstructured fibers (ASMF, also called "holey fibers") [39]-[41].

As discussed earlier, in the context of (13), we saw that in the linear phase-matching regime that the limiting factor for wide operating bandwidth is the fourth-order propagation constant $\beta_{4}$. However, as the impact of the nonlinear phase mismatch $\left(\phi_{\mathrm{NL}}=2 \gamma P_{p} z\right)$ increases, $\beta_{4}$ can be advantageously utilized to increase and flatten the operational bandwidth by optimizing $\beta_{2}$ [7], [42].

A second factor to take into account for the OPA gain bandwidth is the fact that $\lambda_{0}$ in a real fiber is slightly distributed along the fiber length [9], [43], [44]. This will broaden the resulting 


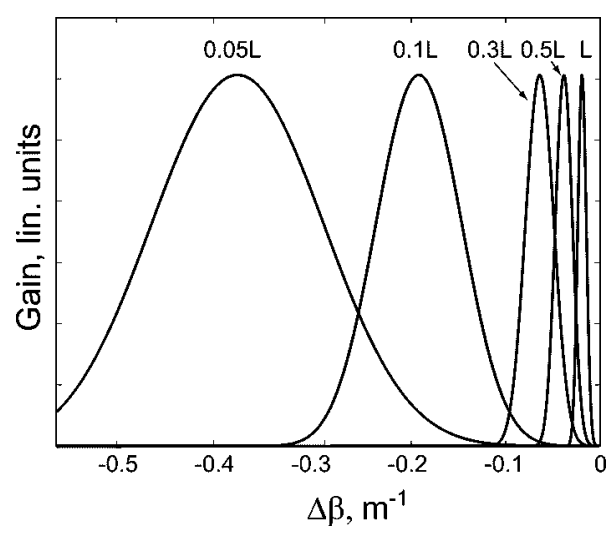

Fig. 5. Calculated single-pass gain versus $\Delta \beta$ for a fiber optical parametric amplifier using five different fiber lengths. The product $\gamma P_{p} L=10$ is fixed for all lengths. The peak gain in each case is $8.7 \times 10-6=81 \mathrm{~dB}$.

gain bandwidth compared to a fixed $\lambda_{0}$ wavelength but will decrease the peak gain. By deliberately introducing a $\lambda_{0}$ variation in the fiber it has been shown that a flat, broad-band operation may be achieved [45]-[48]. A third factor is the existence of birefringence which will decorrelate the polarization of initially copolarized light waves [49], [50].

As the linear phase mismatch $\Delta \beta$ is proportional to the dispersion slope, a fiber with small dispersion slope would increase the signal bandwidth further. Another technique compensating for phase mismatch and allowing to closely design the dispersion profile of the nonlinear medium is quasi-phasematching (QPM). This can be accomplished by periodically inserting short fiber pieces with a sign of $\beta_{2}$ and $\beta_{4}$ compensating for the accumulated phase mismatch [45], [51]. QPM is a well-known technique in the context of $\chi^{(2)}$ nonlinearities in for example periodically pooled $\mathrm{LiNbO}_{3}$ or KTP crystals. In the context of fiber OPA, a problem is the impractical accumulation of optical loses due to the many fiber intersections. One way of avoiding that problem is to implement a fiber grating employing alternating negative and positive dispersion or by using alternating birefringence as demonstrated by Murdoch et al. [52].

A very interesting implementation predicted by Marhic et al. in 1996 [8] and Radic et al. in 2002 [53], demonstrated by Yang et al. [54], Radic et al. [53] and Boggio et al. [55] shows that a flat exponential parametric gain over with a negligible gain ripple may be achieved by using a copolarized dual pump scheme. The dual pumps here are symmetrically positioned around the zero dispersion wavelength enclosing the signal and the idler wavelength, and the wide flat gain bandwidth is generated through a cascaded coupling between nondegenerated and degenerated FPM processes.

Summarizing, key parameters such as high pump power, high nonlinearity coefficient, a short fiber length, a pump wavelength close to the zero-dispersion wavelength, and a low dispersion slope are identified for achieving a high gain and a wide bandwidth in single-pumped fiber optical parametric amplifiers. Recently, Ho et al. demonstrated a fiber-based OPA with more than 200-nm bandwidth using only 20-m HNLF [10]. Using a combination of a short HNLF and parametric gain Westlund $e t$ al. recently demonstrated a transparent fully configurable wavelength converter with $60-\mathrm{nm}$ pump tuning range by filtering out the generated idler wavelength [11].

\section{E. Comparison With Raman Amplifiers}

It is interesting to compare the performance of the OPA with the Raman amplifier, both relying on nonlinear effects in silica fibers but resulting from different phenomena. The gain of phase matched parametric amplification have similarities with Raman amplification such as having an exponential gain dependence on pump power, $P_{p}$ and fiber length, $L$. The gain of the Raman amplifier may be written in decibel units as [56], [57]

$$
G_{R, \mathrm{~dB}}=P_{p} L S_{R}
$$

Here, the Raman gain slope $S_{R} \cong 8.7 g_{R} / A_{\text {eff }}$ where $g_{R}$ is the peak Raman gain coefficient and $A_{\text {eff }}$ is the effective mode area of the fiber. For a fair comparison with the parametric amplifier, we have assumed that the state of polarization between the pump and the signal is aligned throughout the fiber. Table I shows calculated parametric gain slope and Raman gain slope calculated for typical fiber parameters for five different optical fibers [57]-[59]. The OPA has approximately twice as high differential gain as the Raman amplifier for the same fiber parameters in the most cases. This is essentially due to the basic properties of silica and may be traced to the higher value of the nonlinear refractive index, $n_{2}$ compared to $g_{R}$. The higher differential gain makes the OPA more suitable for lumped amplification as well as for all-optical signal processing applications. Both amplifiers have an excitation life time in the order of femtoseconds. This is a necessary requirement for ultrafast signal processing while it is generally considered a drawback in signal amplification applications. Operating a Raman or OPA amplifier in saturated mode will, due to their instantaneous response, degrade the extinction ratio of the signal as the 0 s will be more amplified than the 1s [28]. It will also cause interchannel crosstalk problems in WDM systems. On the other hand, due to the lower efficiency of a Raman amplifier, it is more difficult to saturate than an OPA. The feature of instantaneous saturation has been proposed as a tool for using OPAs as all-optical limiters [30], however, due to the extinction ratio degradation, this is difficult to implement effectively in a system carrying real data. Means of overcoming the extinction ratio problem have been suggested by filtering out a higher order parametric component [60], [61]. Saturation is not a problem for EDFAs which have excitation life time in the order of milliseconds.

An important difference between the two amplifiers is the creation of an idler in the parametric process. In a short HNLF this may be utilized for transparent dynamic wavelength conversion [11]. In pure silica, the Raman gain peaks at a frequency 13.2 $\mathrm{THz}$ below the pump frequency. Due to the exponential gain the 3-dB bandwidth is gain dependent, but is generally $20-30$ $\mathrm{nm}$ wide determined by the vibrational modes of the medium [56]. The bandwidth of the parametric amplifier on the other hand is solely limited by the phase-matching condition between the involved light waves in the optical fiber. In similarity with the OPA, the Raman amplifier requires a phase-matching condition. However, as Raman amplification is due to the scattering between an optical phonon and a photon and the optical phonon has an almost uniform dispersion relation versus wave number [6], [62], the phase matching is easily obtained for arbitrary directions between the pump-and-signal waves. The 
TABLE I

Parametric Gain Slope, $S_{P}$ And Raman Gain Slope, $S_{R}$ For Five Typical Optical Fibers; SMF: Standard Single Mode Fiber, DSF: DisPersion

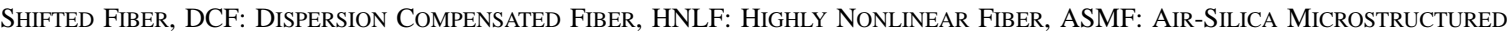
OPTICAL Fiber (“Holey Fiber”). * THE VALUe Is INTERPOLATED From the REDUCED EFFECTIVE AREA

\begin{tabular}{lrrrrr}
\hline Fiber & SMF & DSF & DCF & HNLF & ASMF \\
\hline Attenuation, dB $/ \mathrm{km}$ & 0.2 & 0.2 & 0.45 & 0.7 & $80-240$ \\
Effective area, $A_{\mathrm{eff}}, \mu \mathrm{m}^{2}$ & 85 & 50 & 19 & 12 & 3 \\
Raman gain coefficient, $g_{R} / A_{\mathrm{eff}}, \mathrm{W}^{-1} \mathrm{~km}^{-1}$ & 0.35 & 0.6 & 3 & 7 & $28^{*}$ \\
Nonlinear coefficient at $\mathbf{1 5 5 0 ~ n m ~}, \gamma=n_{2} / A_{\mathrm{eff}}, \mathrm{W}^{-1} \mathrm{~km}^{-1}$ & 1.8 & 2.7 & 5 & 15 & 50 \\
\hline Raman gain slope, $\mathrm{dB} \mathrm{W}^{-1} \mathrm{~km}^{-1}$ & 3 & 5 & 26 & 61 & 244 \\
Parametric gain slope, $\mathrm{dB} \mathrm{W}^{-1} \mathrm{~km}^{-1}$ & 16 & 23 & 44 & 131 & 435
\end{tabular}
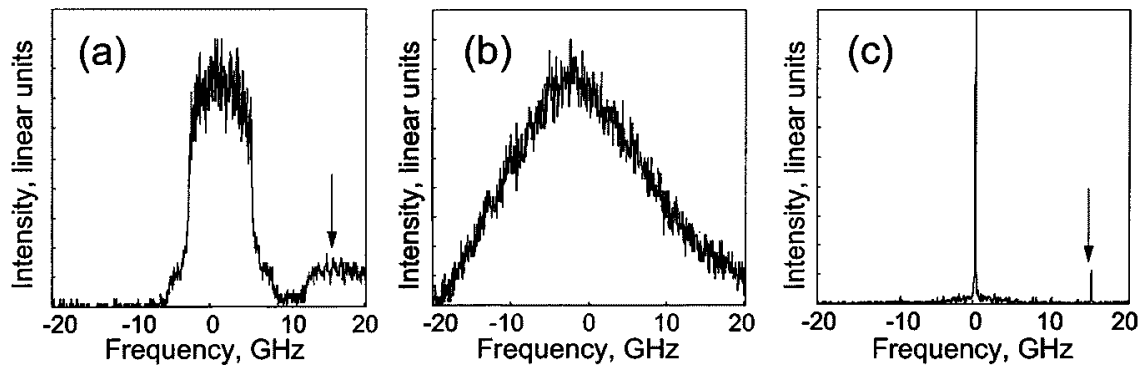

Fig. 6. Measured output spectrum for (a) pump, (b) idler, and (c) amplified signal. Arrows indicate artifacts due to the used Fabry-Pérot etalon.

Raman amplifier can thus use both copropagating and counterpropagating pump schemes contrary to the OPA which can only use copumping. The easily maintained phase-matching condition in combination with the smaller differential gain allows Raman amplifiers to operate as distributed amplifiers over tens of kilometers range. On the other hand, the same easily obtained phase condition prevents the Raman amplifiers from operating in a phase-sensitive mode. As seen in the previous theory section, an OPA operating over long fiber lengths will have a reduced bandwidth. The way to implement a distributed OPA in future optical communication systems would be to use dispersion-flattened fiber, having a fixed dispersion with zero-dispersion slope. The Raman counterpropagating scheme is not only advantageous because it offers distributed amplification, but also from a polarization-sensitive point-of-view. Raman amplification is a polarization-sensitive process, however, by using long fibers and counterpropagating pumping, the states of polarization will evolve in the fiber in such a way that the gain will be reduced by a factor of 2 (in decibel B, e.g., 30 to $15 \mathrm{~dB}$ ), while the polarization dependence of the amplified signal will be significantly reduced. The polarization-sensitive process of parametric amplification is a major obstacle for a possible implementation in commercial optical communication systems. Inoue [63] demonstrates polarization-independent wavelength conversion using FPM by dual pump waves with orthogonal polarization. This scheme implemented in an OPA configuration, i.e., with a nonnegligible nonlinear phase shift was demonstrated by Wong et al. in 2002 [64]. Polarization-independent operation can also be implemented through a single pump using a polarization beam splitter in a Sagnac loop configuration [65]. Although the dual pump configuration is more complex it offers the additional advantage of a flat high-bandwidth gain as discussed above.

\section{APPLICATIONS}

An amplifier operating with a CW pump has several advantages compared to one operating with a pulsed pump; it is fully bit rate transparent, it requires no synchronization, and the pump does not suffer from SPM or induce XPM. The drawbacks are the higher required average pump power and the decreased stimulated Brillouin scattering threshold (SBS) [66], [67]. Although newly developed HNLF offers a higher $\gamma$ and, thus a much shorter required interaction length, the SBS limited maximum power into the fiber will be reduced by the same amount [68]. Thus, methods to increase the SBS threshold are essential for CW pumped OPAs. Proposed methods include broadening of the pump spectrum by PM [69] or arrangements such as strain or temperature distributions [68], [70] to broaden the Brillouin gain bandwidth of the fiber. Drawback of the former is chirping of the idler spectrum as a consequence of the underlying FPM process. Fig. 6 show pump, idler, and signal spectrum after parametric amplification. The spectra are measured using a Fabry-Pérot interferometer; arrows indicate artifacts due to the measurement setup. The pump spectrum is broadened using four combined RF-frequencies (100, 310, 920 , and $2700 \mathrm{MHz}$ ) generating $3^{4}=81$ tones separated 100 $\mathrm{MHz}$ apart. The resulting flat pump spectrum full-width at half-maximum (FWHM) is $8.1 \mathrm{GHz}$ wide. Here, each generated spectral component of the pump spectrum will act as a pump to the signal. From Fig. 1, we may observe that when using a single pump configuration, the generated signal gain $\left(\omega_{221}\right.$ or $\left.\omega_{331}\right)$ will always overlap with the signal under amplification $\left(\omega_{1}\right)$, thus as the frequency of the pump changes over time (due to PM) this will not result in a spectral broadening of the signal spectrum. The generated idler on the other hand will shift its frequency twice as much as the frequency deviation of 


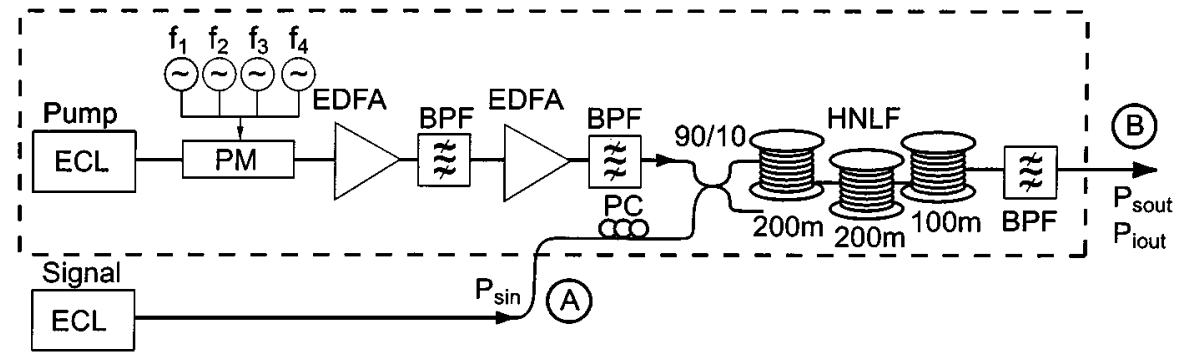

Fig. 7. Experimental setup demonstrating 39-dB CW pumped net "black-box" parametric gain. EDFA: Erbium-doped fiber amplifier. ECL: External cavity laser. PM: Phase modulator. BPF: Optical bandpass filter. OSA: Optical spectrum analyzer. PC: Polarization controller. HNLF: Highly nonlinear fiber.

the pump, thus leading to a broadening of the idler spectrum to twice the spectral width of the phase-modulated pump. Yang et al. proposed in [4] a dual pump scheme using pumps with a relative phase difference of the electrical PM frequency of $\pi$ as a method to compensate for the idler broadening.

\section{A. Linear Optical Amplifier}

Fig. 7 shows the experimental setup for a CW pumped OPA providing 39-dB net "black-box gain" [5]. A CW distributed feedback (DFB) laser diode with a wavelength $2 \mathrm{~nm}$ above $\lambda_{0}$ of the fiber is used as pump source. The pump is PM to broaden its spectrum using four combined sinusoidal RF-frequencies increasing the SBS threshold from 17 to $>33 \mathrm{dBm}$. It is then amplified to approximately $2 \mathrm{~W}$ and combined with the signal using a $90 / 10$ coupler adding $90 \%$ of the pump with $10 \%$ of the signal. The combined pump and signal are coupled into 500-m HNLF with $\gamma=11 \mathrm{~W}^{-1} \cdot \mathrm{km}^{-1}$, the HNLF consisted of three pieces of fiber 200,200 , and $100 \mathrm{~m}$ with a zero dispersion wavelength $\lambda_{0}$, equal to $1556.8,1560.3$, and $1561.2 \mathrm{~nm}$, respectively. The dispersion slope was $0.03 \mathrm{ps} / \mathrm{nm}^{2} \cdot \mathrm{km}$. An optical bandpass filter $(\mathrm{BPF})$ is positioned after the fiber to recover either the generated idler or the amplified signal. Fig. 8 shows an example of the optical spectrum measured at point B in Fig. 7. Here, the optical BPF after the HNLF was removed. Note that the amplified spontaneous noise floor shows the OPA gain profile. Fig. 9 shows measured "black-box" gain (output signal power at point B divided by input signal power at point A), together with calculated gain using (20). The amplifier provided net gain over $>35$ $\mathrm{nm}$ with a peak net gain of $39 \mathrm{~dB}$. The actual fiber gain was 49 $\mathrm{dB}$ due to the 10-dB loss in the pump-signal combiner. The calculated gain takes into account the distribution of the zero distribution wavelength, thereby the less than expected peak gain of $55 \mathrm{~dB}$. Using a uniform zero dispersion wavelength and (23), the theoretical peak gain should be $8.7 \times 1.6 \times 0.5 \times 11-6=70$ $\mathrm{dB}$. Bit-error-rate (BER) measurements before and after amplification indicate a noise figure, after compensation for the 10-dB input coupler loss, in the same range as for conventional EDFAs. For practical implementations, the $10-\mathrm{dB}$ coupler would be replaced by a dichroic coupler to eliminate the loss. The discrepancy between calculated gain and measured gain for the high pump powers are due to depletion of the pump. By filtering out the generated idler, wavelength conversion with inherent gain is obtained. Besides linear amplification and wavelength conversion, a third application with the same scheme is mid-span optical phase conjugation/reamplification [71]. Here, the quality of the optical spectrum for the generated idler is essential. The

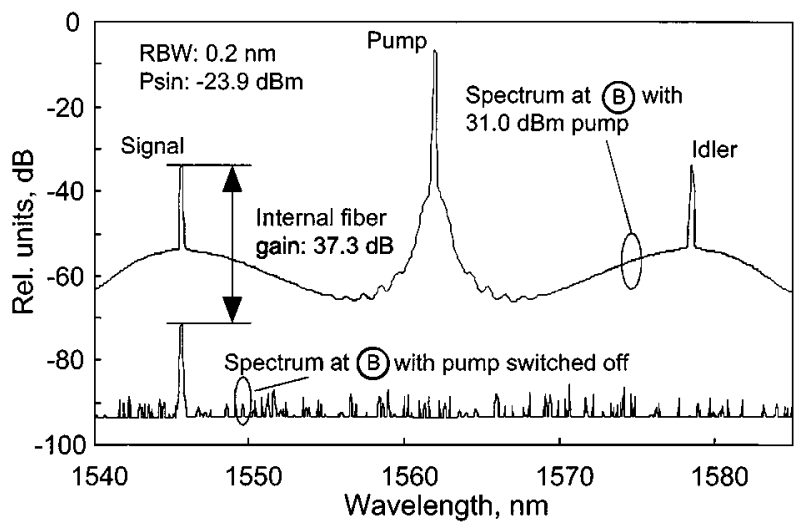

Fig. 8. Optical spectrum measured at OPA output (BPF after HNLF removed)

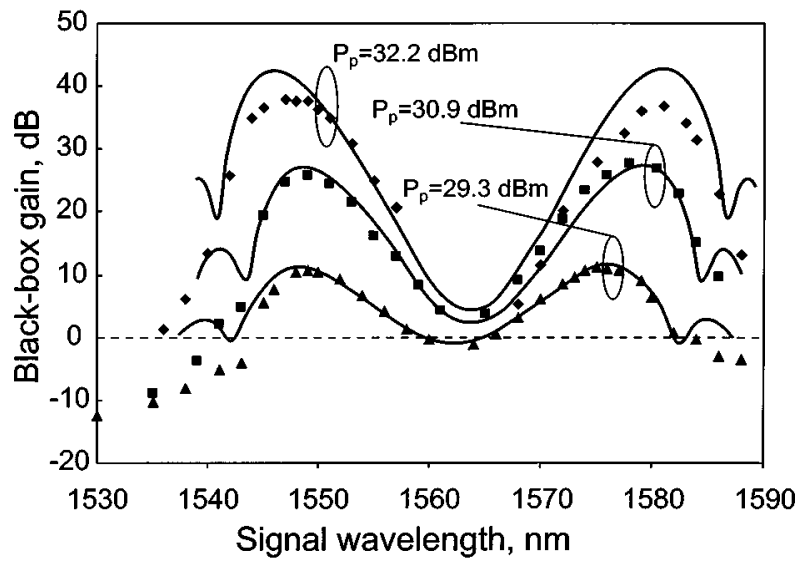

Fig. 9. Measured and calculated net "black-box" gain for three different pump powers $P_{p}$. Note: The given pump power is the actual power coupled into the HNLF.

impact of the idler performance from the PM scheme for SBS suppression needs to be evaluated.

\section{B. Transparent Wavelength Conversion}

Tunable wavelength conversion will be a key component for reconfigurable network nodes in future WDM systems. The CW pumped OPA has attractive features for this application; it is bit rate transparent, it may be designed to provide gain in the conversion process and, thus operate in a lossless transparent mode and it can be designed to operate over a wide wavelength range. The need for tunability of the pump wave was an obstacle for fiber-based wavelength converters for many years. Due to the required interaction lengths and the phase-matching condition, 


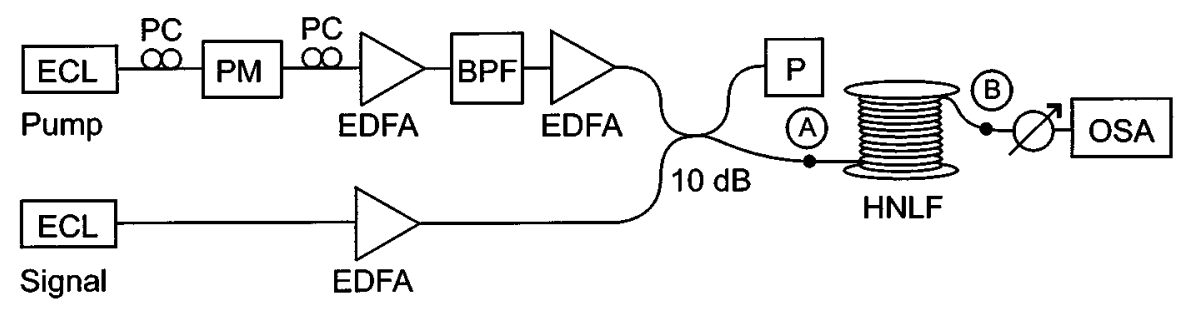

Fig. 10. Experimental setup for transparent wavelength converter. ECL: External cavity laser. PM: Phase modulator. BPF: Optical bandpass filter. OSA: Optical spectrum analyzer. PC: Polarization controller. P: Optical power meter. HNLF: Highly nonlinear fiber.

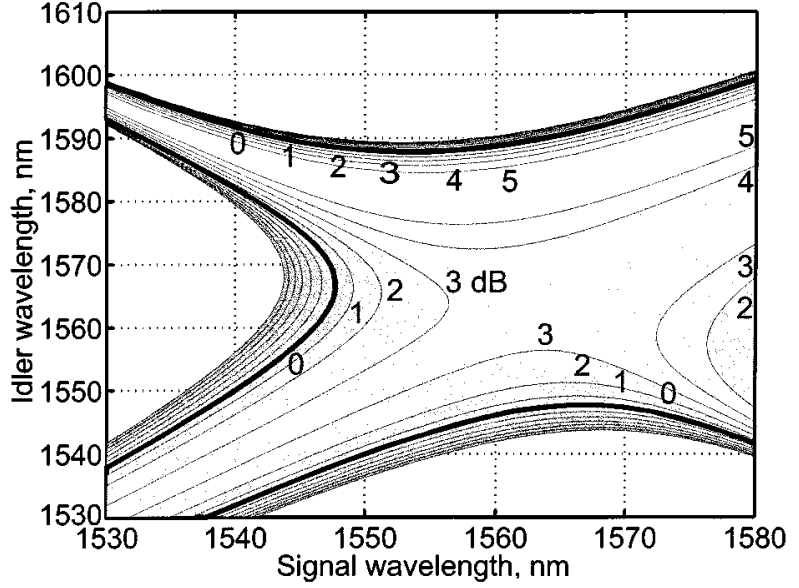

Fig. 11. Contour plot of calculated conversion efficiency versus signal and idler wavelengths. The thick lines show the limit for the transparent $(0 \mathrm{~dB})$ conversion region.

the tuning range of the pump was limited to a few nanometers. This was contrary to the semiconductor optical amplifier (SOA) since the short SOA length offers a wide tuning range for the pump wavelength [72]. With the introduction of new highly nonlinear fibers this advantage is no longer obvious. The keys to a flat wide conversion gain is, as shown in Fig. 5, a short fiber length and a low dispersion slope. Fig. 10 shows the setup for an experiment demonstrating pump tunability over $24 \mathrm{~nm}$ with a conversion bandwidth of $61 \mathrm{~nm}$. A 115-m-long $\mathrm{GeO}_{2}$-doped HNLF with $\gamma=10 \mathrm{~W}^{-1} \cdot \mathrm{km}^{-1}, \lambda_{0}=1559 \mathrm{~nm}$ and dispersion slope of $0.03 \mathrm{ps} / \mathrm{nm}^{2} \cdot \mathrm{km}$ was used. To increase the SBS limited input power with negligible impact on the generated idler, a Brillouin frequency distribution was introduced by winding the HNLF onto eight metal spools, each maintained at different temperatures [68]. In addition, the pump was PM to provide a spectral width of $2.6 \mathrm{GHz}$ resulting in a converted idler spectral width of $5.2 \mathrm{GHz}$. The SBS limited input power was, thus increased to $>33 \mathrm{dBm}$ and the input pump power was fixed to $31 \mathrm{dBm}$. In the experiment, two wavelength-tunable external cavity lasers (ECL) were used, one served as pump source and the second served as signal source. The conversion efficiency was measured with the optical spectrum analyzer by tuning the pump for each signal wavelength. In Fig. 11, the calculated wavelength conversion efficiency $G_{i}$ versus signal wavelength and converted idler wavelength are shown $L=115 \mathrm{~m}, \gamma=10$ $\mathrm{W}^{-1} \cdot \mathrm{km}^{-1}, \lambda_{0}=1561 \mathrm{~nm}$, and $d D / d \lambda=0.03 \mathrm{ps} / \mathrm{nm}^{2} \mathrm{~km}$. For instance, predicted conversion efficiency for converting a WDM channel at 1550 to $1570 \mathrm{~nm}$ should be slightly below 2 $\mathrm{dB}$. In this case, we will use a pump wavelength of $1560 \mathrm{~nm}$. The

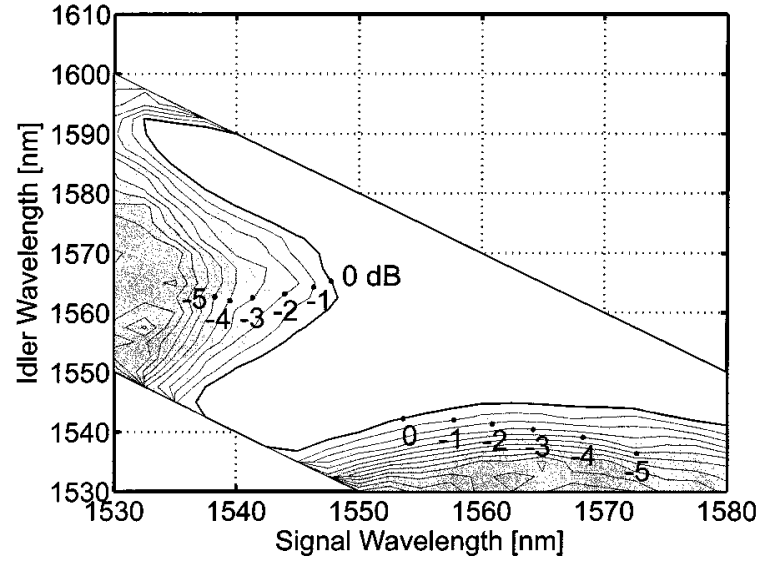

Fig. 12. Contour plot of measured conversion efficiency versus signal and idler wavelengths. Level curves representing a conversion efficiency $<0 \mathrm{~dB}$ are displayed in order to visualize the transparent region.

higher efficiency $(5 \mathrm{~dB})$ for the longer converted wavelengths are due to the reduced phase mismatch parameter $\kappa$ for $\lambda_{p}>\lambda_{0}$. Fig. 12 shows measured conversion efficiency over the available measurement range. Areas in upper right and lower left corners were not reachable due to experimental constrains. The experimental results show good agreement with theory. BER measurements showed $<1.4-\mathrm{dB}$ penalty from the wavelength conversion process.

\section{RZ Pulse Generation}

As was observed in (22) in the previous theory section, when the OPA is operating in a phase-matched condition, it will exhibit an exponential gain dependence on the pump power. Fig. 13(a) shows the normalized gain profile when a sinusoidally intensity modulated pump with a modulation period of one is used. The parameter is the gain slope, $S_{p}$ times the fiber length $L$. Note that the theory used for this figure is simplified and only serves to show the principle of the idea, e.g., the power-dependent nonlinear phase shift is not considered and thus perfect phase matching is considered over the complete gain profile. Fig. 13(b) shows the calculated FWHM width of the gain profile versus $L S_{p}$. For high gain, $G \gg 1$ the parametric amplifier may be approximated with an optical switch, amplifying everything within the FWHM width and being transparent to everything outside. For example, as will be demonstrated below, by using a 40-GHz sinusoidally modulated pump it is possible to generate optical RZ pulses at either the idler or the signal wavelength with a repetition rate of $40 \mathrm{GHz}$ and a pulsewidth as short as 3 ps. For large $L S_{p}$, the window profile approaches a Gaussian. The characteristic 


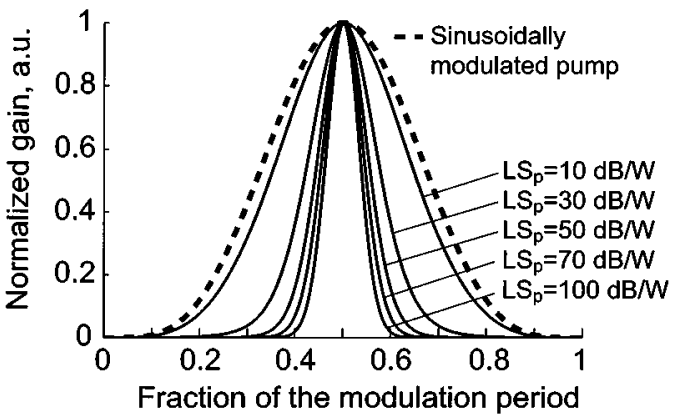

(a)

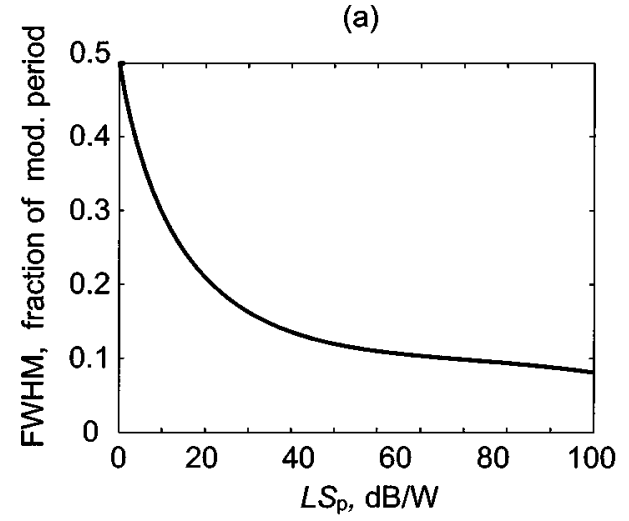

(b)

Fig. 13. (a) Calculated normalized gain time window profile for OPA with a sinusoidally modulated pump versus $L S_{p}, \mathrm{~dB} / \mathrm{W}$. (b) FWHM time window versus $L S_{p}$.

exponential gain may be utilized for several all-optical signal processing applications requiring an ultra high bandwidth. These applications include pulse compression [73] or RZ pulse generation [27], O-TDM switching [29] and an increased resolution in all-optical sampling systems [31]. The following three subsections will provide a more detailed presentation of these applications.

As described above, it should be possible to generate short optical pulses from a sinusoidally modulated pump signal. Fig. 14 shows the setup for an experiment generating $40-\mathrm{GHz}$ RZ pulses from a single frequency sinusoidally amplitude modulated pump. Here, 500-m HNLF is used with $\gamma=11$ $\mathrm{W}^{-1} \cdot \mathrm{km}^{-1}$ and dispersion slope $d D / d \lambda=0.03 \mathrm{ps} / \mathrm{nm}^{2}$ $\mathrm{km}$. The estimated $L S_{p}$ product was, thus $48 \mathrm{~dB} / \mathrm{W}$ which agreed well with the measured $L S_{p}$ product of $50 \mathrm{~dB} / \mathrm{W}$. A Mach-Zehnder intensity modulator (IM) was added in front of the high-power EDFA with a resulting input pump peak power into the fiber of $1.6 \mathrm{~W}$. The principle of operation is to selectively amplify a weak seed CW signal and either recover the signal or the generated idler as the pulse source [27], [74]. Ideally, the extinction ratio in decibels for the pulses on the signal wavelength should be equal to the parametric gain $G=P_{p} L S_{p}-6 \mathrm{~dB}$ for the phase-matched case. By tuning the $\mathrm{CW}$ signal wavelength, the RZ pulse source will shift in carrier wavelength. It is possible to show that the pulse shape approaches a Gaussian as $L S_{p}$ increases [27]. Nearly transform-limited, high power, and very stable $40-\mathrm{GHz}$ pulses at both signal and idler wavelengths with widths between 2 and 4 ps over a 37-nm-wide wavelength range were obtained. Fig. 15(a) shows received $40-\mathrm{GHz}$ pulses detected with a
45-GHz photodetector and a 50-GHz oscilloscope over $5 \mathrm{~min}$. There is no visible amplitude or timing jitter. Fig. 15(b) shows the optical spectrum for the pulses at the signal wavelength with the inset showing a streak camera trace with a resolution of 3.5 ps. By varying the average pump power between $700 \mathrm{~mW}$ and $1.05 \mathrm{~W}$, the average signal output power was tuned between -9 and $+15 \mathrm{dBm}(260-\mathrm{mW}$ peak power) with preserved pulse quality. As the average output power increased above $10 \mathrm{dBm}$ the output pulse width started to increase due to a combined effect of GVD, SPM, and XPM in the HNLF.

\section{O-TDM Switch}

The narrow gain time window profile in pump-modulated OPAs may be utilized in ultrahigh-speed O-TDM switches. From Fig. 13(b), we observe that the product between fiber length and parametric gain slope should be higher than 20 $\mathrm{dB} / \mathrm{W}$ to achieve a 3-dB switching window width smaller than $25 \%$ of the applied sinusoidal modulation period. In [29], we used a 10-GHz sinusoidally modulated pump to demultiplex a 40-Gb/s O-TDM signal. The OPA is demonstrated as a combined demultiplexer, pre-amplifier and, if desired, wavelength converter. The principle is to selectively amplify or wavelength convert a predetermined O-TDM channel. The high gain offers O-TDM demultiplexing with optional wavelength conversion by recovering the selectively amplified signal wavelength. This is in contrary to previously demonstrated FPM-based demultiplexing [75], where wavelength conversion of the demultiplexed signal is unavoidable due to the low efficiency of the FPM-process. The demultiplexer setup is shown in Fig. 16 [29]. The same fiber parameters as in the previously demonstrated RZ-pulse source application are used, resulting in $L S_{p}=48 \mathrm{~dB} / \mathrm{W}$. A switch was inserted in front of the input signal port in order to be able to switch between a $40-\mathrm{Gb} / \mathrm{s}$ $\mathrm{O}-\mathrm{TDM}$ signal and a CW signal generated by a wavelength tunable ECL. The CW signal was used for measuring the resulting FWHM of the switching window by detecting the partially amplified/wavelength converted signal on the digital oscilloscope. Fig. 17 shows measured FWHM switching window widths for idler and signal wavelengths. Fig. 18 shows bit-error rates for all four demultiplexed $10-\mathrm{Gb} / \mathrm{s}$ channels when the OPA was used as a combined $40-$ to $10-\mathrm{Gb} / \mathrm{s}$ demultiplexer/preamplifier to the $10-\mathrm{Gb} / \mathrm{s}$ receiver. The gain was 30 dB. For comparison, the measured back-to-back curve for the thermally limited $10-\mathrm{Gb} / \mathrm{s}$ receiver is also displayed. The lower slope for the preamplified $10-\mathrm{Gb} / \mathrm{s}$ channels compared to the thermally limited $10-\mathrm{Gb} / \mathrm{s}$ back-to-back signal is a well-known property of ASE-limited preamplifiers. When compensating for the 10-dB signal in-coupling loss, the OPA demultiplexer would have a sensitivity at BER $=10^{-9}$ of approximately $-30 \mathrm{dBm}$. The insets show input $40-\mathrm{Gb} / \mathrm{s}$ O-TDM data and demultiplexed $10-\mathrm{Gb} / \mathrm{s}$ data.

\section{E. All-Optical Sampling}

Direct monitoring of optical signals will be essential for future ultrahigh-speed communication systems [76]. All-optical sampling is a technique that enables real-time evaluation of a received high bit rate signal. All-optical sampling based on parametric amplification is a similar application to the O-TDM 


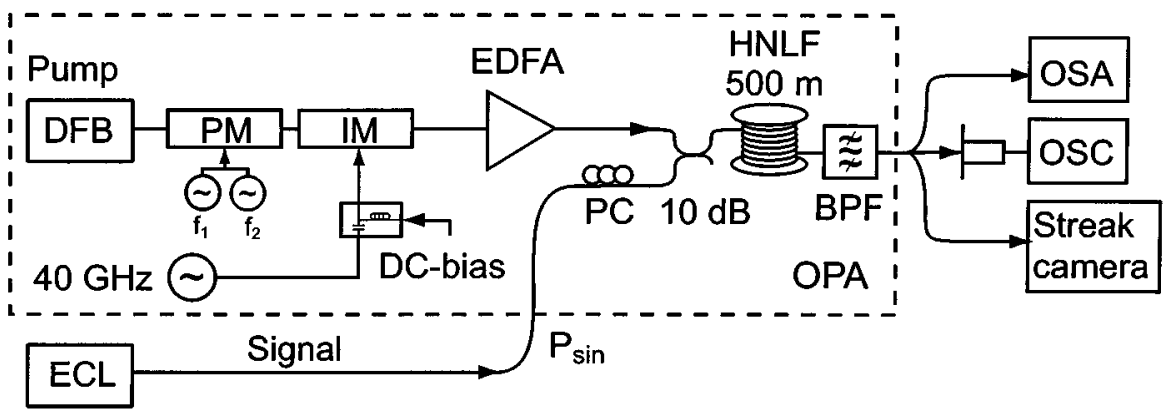

Fig. 14. Experimental setup for 40-GHz RZ pulse source. ECL: External cavity laser. DFB: Distributed feedback laser diode. EDFA: Erbium-doped fiber amplifier, PM: phase modulator. IM: Mach-Zehnder intensity modulator. BPF: Optical bandpass filter. OSA: Optical spectrum analyzer. OSC: Digital oscilloscope. PC: Polarization controller. HNLF: Highly nonlinear fiber.

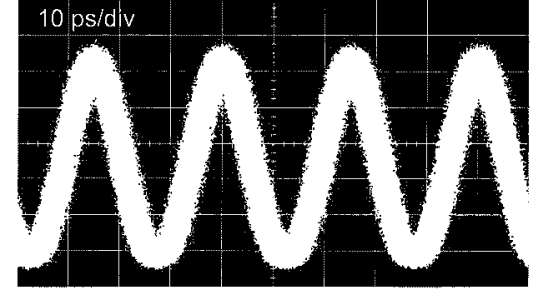

(a)

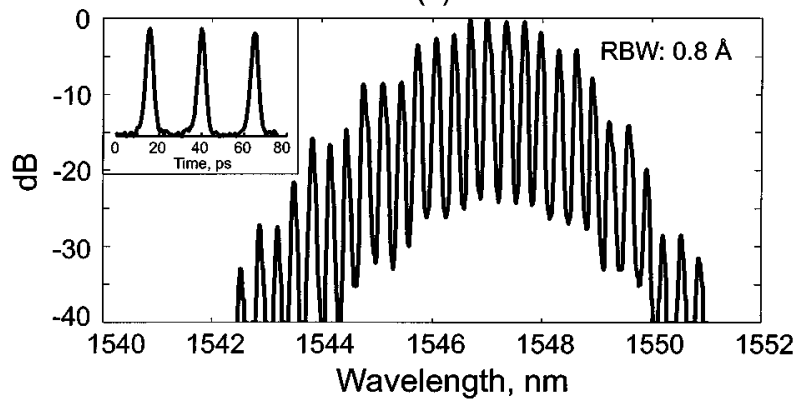

(b)

Fig. 15. OPA generated $40-\mathrm{GHz}$ pulses. (a) Signal waveform over 5 min detected by a $45-\mathrm{GHz}$ photodetector and an oscilloscope with $50-\mathrm{GHz}$ bandwidth. (b) Optical spectrum. The inset shows a streak camera trace of a generated $40-\mathrm{GHz}$ pulse train (3.5-ps resolution).

switch. Until recently, optical fibers were not considered a serious alternative for such applications due to the small nonlinear coefficient. Instead, $\chi^{(2)}$ sum-frequency-generation in nonlinear crystals has generally been used [77], [78]. In [31], an all-optical sampling application using fiber-based parametric amplification is described. The setup is shown in Fig. 19. The data signal under study is a bursted O-TDM signal at either 160,200 , or $300 \mathrm{~Gb} / \mathrm{s}$. The sampling system uses a mode-locked Erbium-doped fiber ring laser (ML-EFRL) generating 1.6-ps-wide pulses at the original 10-Gb/s O-TDM channel repetition frequency plus a slight deviation $\Delta f$. The frequency deviation $\Delta f$ is usually in the kilohertz range. An intensity modulator removes most pulses so that 1.6-ps FWHM sampling pulses at $\approx 100-\mathrm{MHz}$ repetition rate $(7-\mathrm{W}$ peak power) are generated and subsequently combined with the data signal to be studied. The nonlinear medium consists of $50-\mathrm{m}$ HNLF with $\lambda_{0}=1557 \mathrm{~nm}$ and $d D / d \lambda=0.03 \mathrm{ps} / \mathrm{nm}^{2} \mathrm{~km}$. The generated idler pulse is detected by a photo detector with $125-\mathrm{MHz}$ bandwidth. The idler envelope which will be a time resolved copy of the input signal, is recorded via a commercial
A/D converter (Gage Compuscope 8500) and a conventional 400-MHz desktop computer. Fig. 20 shows sampled 160-, 200-, and 300-Gb/s eyes. Parametric gain is achieved over more than $30 \mathrm{~nm}$ with at least 20-dB signal-to-noise-ratio for a minimum input signal peak power of $10 \mathrm{~mW}$. The dynamic range was 10 to $500 \mathrm{~mW}$, the higher input level limited by the input signal becoming distorted by SPM. The sampled signal (25000 samples) are visualized in real-time with a refresh rate of $5 \mathrm{~Hz}$. A second feature of this application, not necessarily connected to parametric amplification, is that due to the many samples taken under a very short time period $(<0.2 \mathrm{ps})$ there is no requirement for clock recovery of the visualized signal.

\section{DisCUSSION AND CONCLUSION}

Although the fiber-based CW operated OPA is a well-known technique, its impact in optical communication systems is still relatively unknown. Therefore, it is fair to state that its prospects in practical applications are still unclear, although they appear very promising. Their main advantages are the built-in multifunctionalities and their ability to operate over arbitrarily centered and wide wavelength ranges. While we have here presented some examples of applications, several more are likely to be proposed and demonstrated in the future. To cover extremely wideband WDM applications, amplifiers such as fiber OPAs and/or Raman (lumped and/or distributed) will be needed to amplify all WDM channels simultaneously. Thus, while the EDFA, is a remarkably enabling device, it may not be sufficient for some future applications.

One significantly unique feature of the OPA is the feasibility to achieve noise-free optical amplification, i.e., a 0-dB noise figure. With such amplifiers widely available, the impact on lightwave system design would likely be of the same magnitude as the impact from the introduction of EDFAs. Noiseless optical amplification would mean no SNR degradation along the transmission path. This would essentially lead to the elimination of the impairments caused by fiber nonlinearities (e.g., FPM, SPM, XPM, nonlinear WDM crosstalk, etc.) and, thus also eliminate the need for distributed Raman amplification. The spectral efficiency would increase, as WDM channels could be placed closer to each other. However, the implementation of such an optical phase-sensitive amplifier is very challenging, similar to the coherent homodyne lightwave receivers widely studied in the 1980s. It is clear that significant progress in this area is needed 


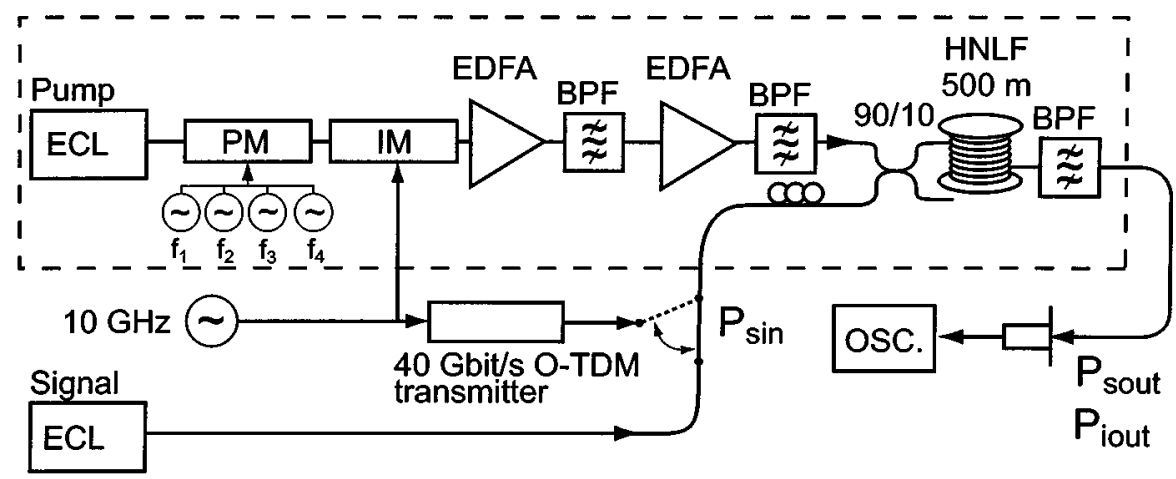

Fig. 16. Experimental setup for a 40- to 10-Gb/s OPA-based time demultiplexer. ECL: External cavity laser. EDFA: Erbium-doped fiber amplifier. PM: Phase modulator. IM: Mach-Zehnder intensity modulator. BPF: Optical bandpass filter. OSA: Optical spectrum analyzer. OSC: Digital oscilloscope. PC: Polarization controller. HNLF: Highly nonlinear fiber.

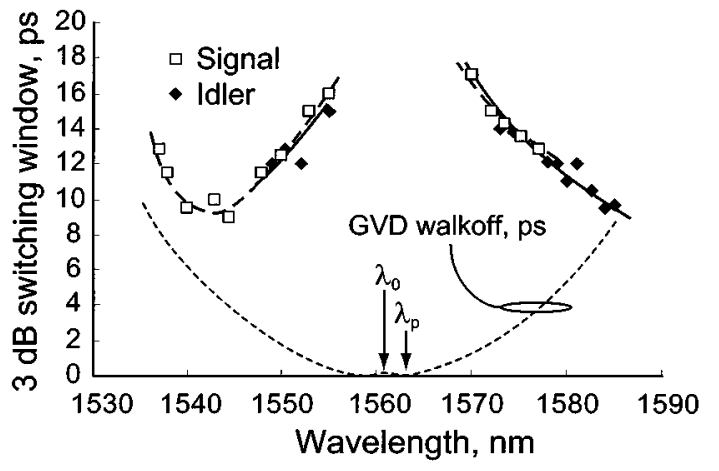

Fig. 17. Measured switching window width versus signal and idler wavelengths. Dashed line: Calculated walkoff due to GVD.

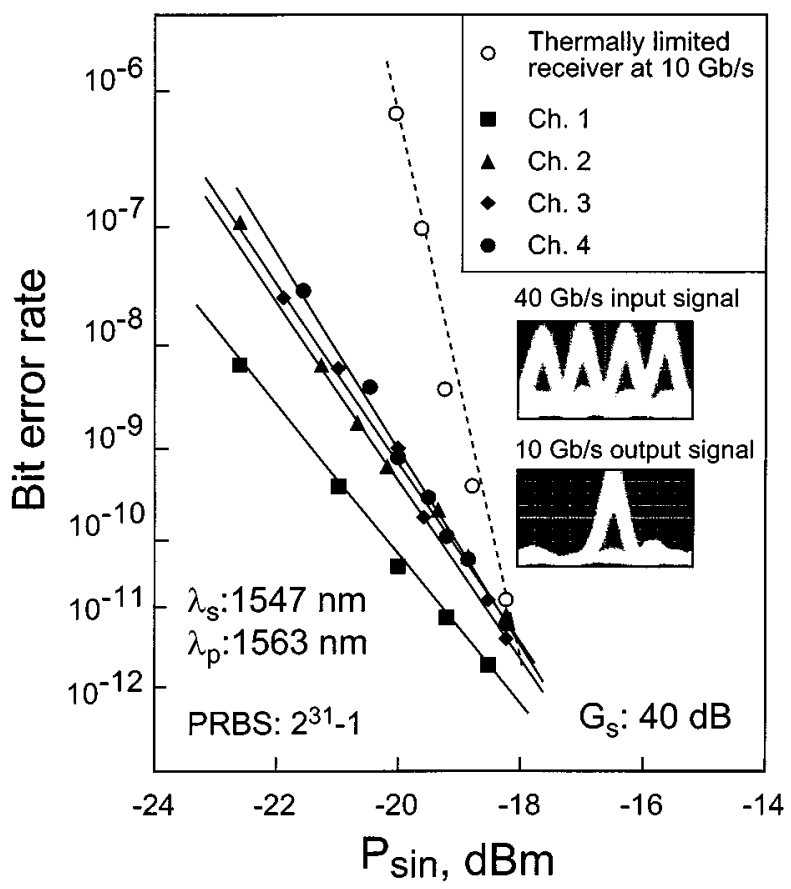

Fig. 18. Measured BER for 40- to 10-Gb/s O-TDM demultiplexing. The insets show $40-\mathrm{Gb} / \mathrm{s}$ input data and $10-\mathrm{Gb} / \mathrm{s}$ demultiplexed output data.

before this would be of practical interest. Meanwhile, another approach to address the same issue (but in a fundamentally dif- ferent way), that is being studied fairly extensively, is all-optical $3 \mathrm{R}$ regeneration which serves to restore the signal integrity. This implementation could involve the use of an OPA, here in the phase-insensitive implementation. The OPA operated in the gain-compressed mode would act as an optical limiter to suppress noise accumulation in the time domain.

From a practical viewpoint, there are a few important issues which need further attention. Polarization dependence is intrinsic to the FPM process. Many applications require polarization-independent operation (although a viable option in many cases may be to rely on polarization-tracking schemes) and practical techniques to solve this are needed, e.g., based on polarization-diversity or dual-pump implementations. Another concern is gain saturation. If an OPA operates such that the pump power is being depleted due to the signal presence, signal degradation may appear both in single-channel systems (e.g., extinction-ratio degradation) and in WDM systems (nonlinear crosstalk). The quantified consequences of this are yet not fully investigated. Yet another issue is the implementation of very wideband and flat gain spectra using multiple pumps, ideally also resulting in polarization independence. Much knowledge can be gained from the research being conducted on the Raman amplifier counterpart.

The recent progress of fiber-based OPAs stems from the development of highly nonlinear single-mode fibers and the availability of high-power semiconductor lasers. Future improvements can be expected from further progress in this area. Specifically, holey fibers appear very promising for this purpose. Challenges here include the splicing of these fibers, reducing the fiber loss and the polarization-mode dispersion, as well as the tailoring of the dispersion profile.

In conclusion, we have reviewed the basic theoretical framework as well as several applications of fiber-based OPAs. The multifunctional features of the OPAs appear particularly promising. We have also discussed some similarities and dissimilarities with Raman amplifiers. The most obvious unique features of the OPAs are the idler generation (useful e.g., for wavelength conversion) and the feasibility of noiseless amplification. However, more work is needed to fully understand the limitations and opportunities of using fiber OPAs in optical lightwave systems. 


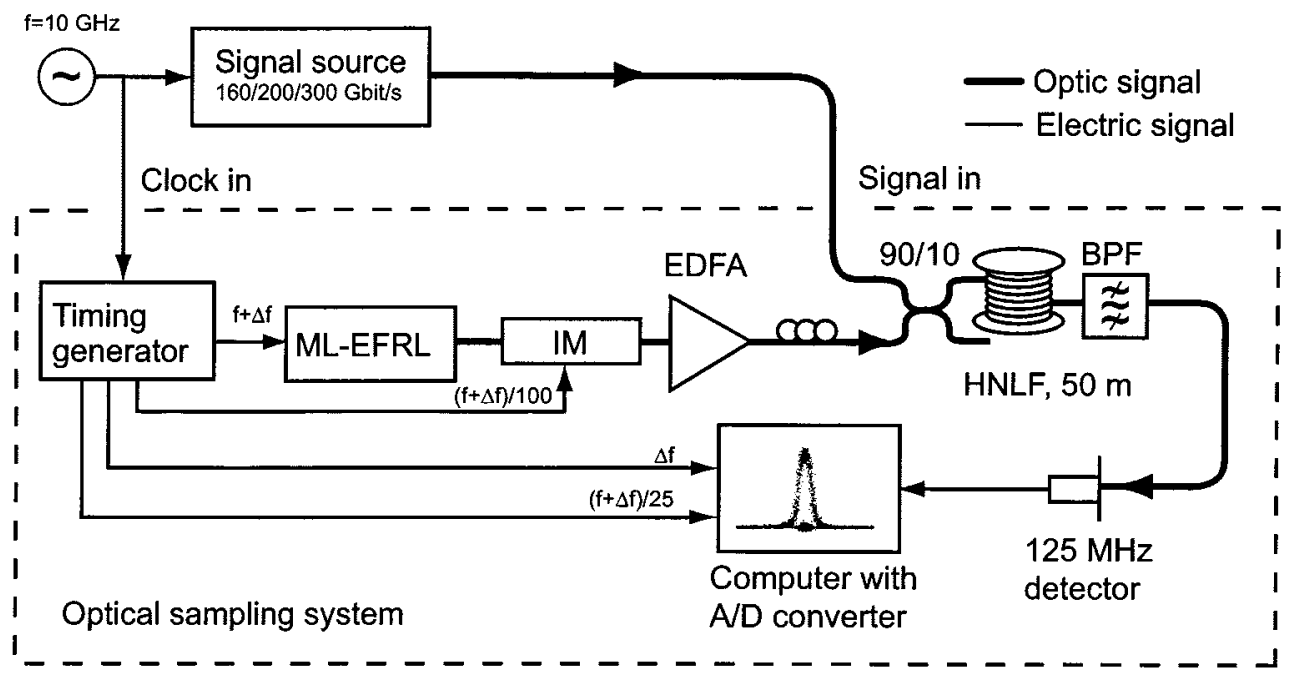

Fig. 19. Experimental setup for an optical sampling system based on parametric amplification. ML-EFRL: 10-GHz actively mode-locked Erbium fiber ring laser. EDFA: Erbium-doped fiber amplifier. IM: Mach-Zehnder intensity modulator. BPF: Optical bandpass filter. OSA: Optical spectrum analyzer. HNLF: Highly nonlinear fiber.
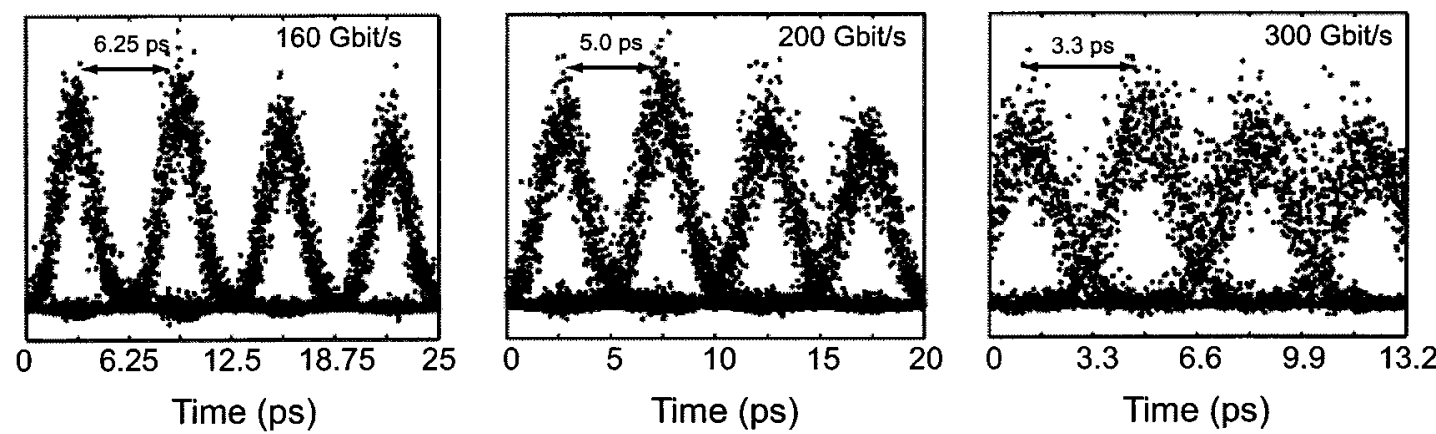

Fig. 20. Sampled eye diagrams of 160-, 200-, and 300- Gb/s optical signals.

\section{ACKNOWLEDGMENT}

The authors would like to acknowledge Sumitomo Electric Industries and OFS Fitel, Denmark for providing the HNLF.

\section{REFERENCES}

[1] S.-K. Choi, R.-D. Li, C. Kim, and P. Kumar, "Traveling-wave optical parametric amplifier: Investigation of its phase-sensitive and phase-insensitive gain response," J. Opt. Soc. Amer. B, vol. 14, pp. 1564-1575, 1997.

[2] M. Onishi, T. Okuno, T. Kashiwida, S. Ishikawa, N. Akasaka, and M. Nishimura, "Higly nonlinear dispersion-shifted fibers and their application to broadband wavelength converter," Opt. Fiber Technol., vol. 4, pp. 204-214, 1998.

[3] D. L. Philen, D. W. Peckham, and I. Brener, "Measurement of the nonlinear index of refraction, $N_{2}$, for various fiber types," in Proc. Optical Fiber Communication Conf., vol. 4, Baltimore, MD, 2000, pp. 184-186. Paper ThL5.

[4] F. S. Yang, M. E. Marhic, and L. G. Kazovsky, "CW fiber optical parametric amplifier with net gain and wavelength conversion efficiency $>1$," Electron. Lett., vol. 32, pp. 2336-2338, 1996.

[5] J. Hansryd and P. A. Andrekson, "Broad-band continuous-wave-pumped fiber optical parametric amplifier with 49-dB gain and wavelength-conversion efficiency," IEEE Photon. Technol. Lett., vol. 13, pp. 194-196, Mar. 2001.

[6] S. Namiki and Y. Emori, "Ultrabroad-band raman amplifiers pumped and gain-equalized by wavelength-division-multiplexed high-power laser diodes," IEEE Select. Topics Quantum Electron., vol. 7, pp. 3-16, Jan.-Feb. 2001
[7] M. E. Marhic, N. Kagi, T.-K. Chiang, and L. G. Kazovsky, "Broadband fiber optical parametric amplifiers," Opt. Lett., vol. 21, pp. 573-575, 1996.

[8] M. E. Marhic, Y. Park, F. S. Yang, and L. G. Kazovsky, "Broadband fiber-optical parametric amplifiers and wavelength converters with lowripple Chebyshev gain spectra," Opt. Lett., vol. 21, pp. 1354-1356, 1996.

[9] M. Karlsson, "Four-wave mixing in fibers with randomly varying zerodispersion wavelength," J. Opt. Soc. Amer. B, vol. 15, pp. 2269-2275, 1998.

[10] M.-C. Ho, K. Uesaka, M. E. Marhic, Y. Akasaka, and L. G. Kazovsky, "200-nm-bandwidth fiber optical amplifier combining parametric and raman gain," J. Lightwave Technol., vol. 19, pp. 977-981, July 2001.

[11] M. Westlund, J. Hansryd, P. A. Andrekson, and S. N. Knudsen, "Transparent wavelength conversion in fiber with $24 \mathrm{~nm}$ pump tuning range," Electron. Lett., vol. 38, pp. 85-86, 2002.

[12] H. Itoh, G. M. Davis, and S. Sudo, "Continuous-wave-pumped modulational instability in an optical fiber," Opt. Lett., vol. 14, pp. 1368-1370, 1989.

[13] R. H. Stolen and J. E. Bjorkholm, "Parametric amplification and frequency conversion in optical fibers," IEEE J. Quantum Electron., vol. QE-18, pp. 1062-1072, 1982.

[14] G. Cappellini and S. Trillo, "Third order three-wave mixing in single-mode fibers: Exact solutions and spatial instability effects," $J$. Opt. Soc. Amer. B, vol. 8, pp. 824-838, 1991.

[15] N. Shibata, R. P. Braun, and R. G. Waarts, "Phase-mismatch dependence of efficiency of wave generation through four-wave mixing in a single-mode optical fiber," IEEE J. Quantum Electron., vol. 23, pp. 1205-1210, June 1987.

[16] G. P. Agrawal, Nonlinear Fiber Optics, 2nd ed. San Diego, CA, USA: Academic, 1995, ch. 10.

[17] W. Imajuku and A. Takada, "Gain characteristics of coherent optical amplifiers using a Mach-Zehnder interferometer with Kerr media," IEEE J. Quantum Electron., vol. 35, pp. 1657-1665, Nov. 1999. 
[18] J. A. Levenson, I. Abram, Th. Rivera, and P. Grangier, "Reduction of quantum noise in optical parametric amplification," J. Opt. Soc. Amer. $B$, vol. 10, pp. 2233-2238, 1993.

[19] R.-D. Li, P. Kumar, and W. L. Kath, "Dispersion compensation with phase-sensitive optical amplifiers," J. Lightwave Technol., vol. 12, pp. 541-549, Mar. 1994.

[20] W. Imajuku and A. Takada, "Pulse form reshaping of chirped pulse using optical phase-sensitive amplifier," in Proc. Optical Fiber Communication Conf., San Diego, CA, 1999, pp. 131-133.

[21] J. N. Kutz, W. L. Kath, R.-D. Li, and P. Kumar, "Long-distance pulse propagation in nonlinear optical fibers by using periodically spaced parametric amplifiers," Opt. Lett., vol. 18, pp. 802-804, 1993.

[22] I. H. Deutsch and I. Abram, "Reduction of quantum noise in soliton propagation by phase-sensitive amplification," J. Opt. Soc. Amer. B, vol. 11, p. 2303, 1994

[23] W. L. Kath, A. Mecozzi, P. Kumar, and C. G. Goedde, "Long-term storage of a soliton bit stream using phase-sensitive amplification: Effects of soliton-soliton interactions and quantum noise," Opt. Commun., vol. 157, pp. 310-326, 1998.

[24] W. Imajuku and A. Takada, "Theoretical analysis of system limitation for AM-DD/NRZ optical transmission systems using in-line phase-sensitive amplifiers," J. Lightwave Technol., vol. 16, pp. 1158-1170, July 1998.

[25] W. Imajuku, A. Takada, and Y. Yamabayashi, "Inline coherent optical amplifier with noise figure lower than $3 \mathrm{~dB}$ quantum limit," Electron. Lett., vol. 36, pp. 63-64, 2000.

[26] M. E. Marhic, C. H. Hsia, and J.-M. Jeong, "Optical amplification in a nonlinear fiber interferometer,” Electron. Lett., vol. 27, pp. 210-211, 1991.

[27] J. Hansryd and P. A. Andrekson, "Wavelength tunable $40 \mathrm{GHz}$ pulse source based on fiber optical parametric amplifier," Electron. Lett., vol. 37, pp. 584-585, Apr. 2001.

[28] P. O. Hedekvist, M. Karlsson, and P. A. Andrekson, "Fiber four-wave mixing demultiplexing with inherent parametric amplification," J. Lightwave Technol., vol. 15, pp. 2051-2058, Nov. 1997.

[29] J. Hansryd and P. A. Andrekson, "O-TDM demultiplexer with $40 \mathrm{~dB}$ gain based on a fiber optical parametric amplifier," IEEE Photon. Technol. Lett., vol. 13, pp. 732-734, July 2001.

[30] W. Imajuku, A. Takada, and Y. Yamabayashi, "Amplitude noise suppression using a high gain phase sensitive amplifier as a limiting amplifier," Electron. Lett., vol. 32, pp. 677-679, 1996.

[31] J. Li, J. Hansryd, P.-O. Hedekvist, P. A. Andrekson, and S. N. Knudsen, "300 Gbit/s eye-diagram measurement by optical sampling using fiber based parametric amplification," Optical Fiber Communication Conf. and Exhibit, vol. 4, 2001.

[32] G. P. Agrawal, Nonlinear Fiber Optics, 2nd ed. San Diego, CA: Academic, 1995.

[33] P. O. Hedekvist, "Optical Phase Conjugation and All-Optical Demultiplexing Using Four-Wave Mixing in Dispersion Shifted Fibers," Ph.D. Thesis, School of Electrical and Computer Engineering, Chalmers University of Technology, Göteborg, Sweden, 1998.

[34] M. O. van Deventer, Fundamentals of Bidirectional Transmission Over a Single Optical Fiber, Leidschendam, The Netherlands: PTT Research, 1994.

[35] G. P. Agrawal, Nonlinear Fiber Optics, 2nd ed. San Diego, CA: Academic, 1995, ch. 2.

[36] A. Vatarescu, "Light conversion in nonlinear single-mode optical fibers," J. Lightwave Technol., vol. 5, pp. 1652-1659, 1987.

[37] K. Inoue and T. Mukai, "Signal wavelength dependence of gain saturation in a fiber optical parametric amplifier," Opt. Lett., vol. 26, pp. $10-12,2001$.

[38] A. R. Chraplyvy, "Limitations on lightwave communications imposed by optical-fiber nonlinearities," J. Lightwave Technol., vol. 8, pp. $1548-1557$, Oct. 1990.

[39] J. Broeng, D. Mogilevstev, S. E. Barkou, and A. Bjarklev, "Photonic crystal fibers: A new class of optical waveguides," Opt. Fiber Technol., vol. 5, pp. 305-330, 1999.

[40] P. St. J. Russell, J. C. Knight, T. A. Birks, S. J. Mangan, and W. J Wadsworth, "Recent progress in photonic crystal fibers," in Proc. Optical Fiber Communication Conf., vol. 3, Baltimore, MD, 2000, Paper ThG1.

[41] X. Liu, C. Xu, W. H. Knox, J. K. Chandalia, B. J. Eggleton, S. G Kosinski, and R. S. Windeler, "Soliton self-frequency shift in a short tapered air silica microstructure fiber," Opt. Lett., vol. 26, pp. 358-360, 2001.
[42] M. Yu, C. J. McKinstrie, and G. P. Agrawal, "Modulation instabilities in dispersion-flattened fibers," Phys. Rev. E, vol. 52, no. 1, pp. 1072-1080, 1995 .

[43] H. Onaka, K. Otsuka, H. Miyata, and T. Chikama, "Measuring the longitudinal distribution of four-wave mixing efficiency in dispersion-shifted fibers," IEEE Photon. Technol. Lett., vol. 6, pp. 1454-1456, Dec. 1994

[44] L. F. Mollenauer, P. V. Mamyshev, and M. J. Neubelt, "Method for facile and accurate measurement of optical fiber dispersion maps," Opt. Lett., vol. 21, pp. 1724-1726, 1996.

[45] K. Inoue, "Arrangement of fiber pieces for a wide wavelength conversion range by fiber four-wave mixing," Opt. Lett., vol. 19, pp. 1189-1191, 1994

[46] Y. Su, L. Wang, A. Agarwal, and P. Kumar, "All-optical limiter using gain flattened fiber parametric amplifier," Electron. Lett., vol. 36, pp. 1103-1104, 2000.

[47] L. Provino, A. Mussot, E. Lantz, T. Sylvestre, and H. Mailotte, "Broadband and flat parametric gain with a single low-power pump in a multisection fiber arrangement," in Proc. Optical Fiber Communication Conference, CA, 2002.

[48] S. E. French and J. L. Blows, "Continous wave optical fiber parametric amplifier with flattened gain," presented at the Optical Amplifiers and Their Applications, Stresa, Italy, 2001, Postdeadline Paper PD7.

[49] R. M. Jopson and R. E. Tench, "Polarization-independent phase conjugation of lightwave signals," Electron. Lett., vol. 29, pp. 2216-2217, 1993.

[50] O. Aso, Y. Tashiro, M. Tadakuma, and S. Namiki, "Effect of modulation instability on broadband wavelength conversion using four-wave mixing in optical fiber," in Proc. Optical Fiber Communication Conf., vol. 3, Baltimore, MD, 2000, pp. 187-189. paper ThL6.

[51] M. E. Marhic, F. S. Yang, M. C. Ho, and L. G. Kazovsky, "High-nonlinearity fiber optical parametric amplifier with periodic dispersion compensation," J. Lightwave Technol., vol. 17, pp. 210-215, Feb. 1999.

[52] S. G. Murdoch, R. Leonhardt, J. D. Harvey, and T. A. B. Kennedy, "Quasiphase matching in an optical fiber with periodic birefringence," J. Opt. Soc. Amer. B, vol. 14, pp. 1816-1822, 1997.

[53] S. Radic, C. J. McKinstrie, A. R. Chraplyvy, G. Raybon, J. C. Centanni, C. G. Jorgensen, K. Brar, and C. Headley, "New class of continous wave parametric amplifiers," in Proc. Optical Fiber Communication Conf., CA, 2002, Postdeadline Paper FB5.

[54] F. S. Yang, M. C. Ho, M. E. Marhic, and L. G. Kazovsky, "Demonstration of two-pump fiber optical parametric amplification," Electron. Lett. vol. 33, pp. 812-813, 1997.

[55] J. M. Boggio, F. A. Callegari, S. Tenenbaum, H. L. Fragnito, J. B. Rosolem, and M. R. X. deBarros, "Demonstration of $26 \mathrm{~dB}$ on-off gain of a two-pump fiber optical parametric amplifier," in Proc. Optical Fiber Communication Conf., CA, 2002, Paper ThGG18, pp. 636-637.

[56] G. P. Agrawal, Nonlinear Fiber Optics, 2nd ed. San Diego, CA: Academic, 1995, ch. 8

[57] T. Okuno, T. Tsuzaki, M. Hirano, T. Miyamotoa, M. Kakui, M. Onishi, Y. Nakai, and M. Nishimura, "Nonlinear-fiber-based discrete Raman amplifier with sufficiently suppressed degradation of WDM signal quality," in Proc. Optical Amplifiers and Their Applications, Stresa, Italy, 2001

[58] H. Kubota, K. Suzuki, S. Kawanishi, M. Nakazawa, M. Tanaka, and M. Fujita, "Low-loss, $2 \mathrm{~km}$-long photonic crystal fiber with zero GVD in the near ir suitable for picosecond pulse propagation at the $800 \mathrm{~nm}$ band," in Tech. Dig. CLEO, vol. 35, 2001, pp. 635-636.

[59] P. J. Bennett, T. M. Monro, and D. J. Richardson, "Toward practical holeyfiber technology: Fabrication, splicing, modeling, and characterization," Opt. Lett., vol. 24, pp. 1203-1205, 1999.

[60] E. Ciaramella, "A new scheme for all-optical signal reshaping based on wavelength conversion in optical fibers," in Proc. Optical Fiber Communication Conf., vol. 2, Baltimore, MD, 2000, Paper WM37, pp. 320-322.

[61] K. Inoue, "Suppression of level fluctuation without extinction ratio degradation based on output saturation in higher order optical parametric interaction in fiber," IEEE Photon. Technol. Lett., vol. 13, pp. 338-340, Apr. 2001.

[62] P. R. Trischitta and E. Varma, Theory of Optical Waveguides, Corona, Tokyo, Japan, 1992, pp. 191-198.

[63] K. Inoue, "Polarization independent wavelength conversion using fiber four-wave mixing with two orthogonal pump lights of different frequencies," J. Lightwave Technol., vol. 12, pp. 1916-1920, Nov. 1994.

[64] K. K. Y. Wong, M. E. Marhic, K. Uesaka, and L. G. Kazovsky, "Polarization-independent and flat-gain $\mathrm{CW}$ two-pump fiber optical parametric amplifier and wavelength converter," in Proc. Optical Fiber Communication Conf., CA, 2002, Paper TuS5, pp. 129-130. 
[65] — " "Polarization-independent fiber optical parametric amplifier," presented at the OECC, Sydney, NSW, Australia, 2001.

[66] R. G. Smith, "Optical power handling capacity of low loss optical fibers as determined by stimulated Raman and Brillouin scattering," Appl. Opt., vol. 11, no. 11, pp. 2489-2494, 1972.

[67] G. P. Agrawal, Nonlinear Fiber Optics, 2nd ed. San Diego, CA: Academic, 1995, ch. 9.

[68] J. Hansryd, F. Dross, M. Westlund, P. A. Andrekson, and S. N. Knudsen, "Increase of the SBS threshold in a short highly nonlinear fiber by applying a temperature distribution," J. Lightwave Technol., vol. 19, pp. 1691-1697, Nov. 2001.

[69] S. K. Korotky, P. B. Hansen, L. Eskildsen, and J. J. Veselka, "Efficient phase modulation scheme for suppressing stimulated Brillouin scattering," in Tech. Dig. International Conf. Integrated Optics and Optical Fiber Communications, vol. 2, Hong Kong, 1995, Paper WD2, pp. $110-111$

[70] K. Shiraki, M. Ohashi, and M. Tateda, "SBS threshold of a fiber with a Brillouin frequency shift distribution," J. Lightwave Technol., vol. 14, pp. 50-56, Jan. 1996.

[71] S. Watanabe, S. Kaneko, and T. Chikama, "Long-haul fiber transmission using optical phase conjugation," Opt. Fiber Technol., vol. 2, pp. 169-178, 1996.

[72] G. Hunziker, R. Paiella, A. D’Ottavi, P. Spano, R. Dall'ara, G. Guekos, and $\mathrm{K}$. Vahala, "30 $\mathrm{nm}$ wavelength conversion at $10 \mathrm{Gbit} / \mathrm{s}$ by four-wave mixing in a semiconductor optical amplifier," in Proc. Optical Fiber Communication Conf., San Diego, CA, 1998, Paper WB7, pp. 108-109.

[73] T. Yamamoto and M. Nakazawa, "Active optical pulse compression with a gain of $29.0 \mathrm{~dB}$ by using four-wave mixing in an optical fiber," IEEE Photon. Technol. Lett., vol. 9, pp. 1595-1597, Dec. 1997.

[74] A. T. Clausen, L. Oxenløwe, C. Peucheret, H. N. Poulsen, P. Jeppesen, S. N. Knudsen, and L. Gruner-Nielsen, " $10-\mathrm{GHz}$ return-to-zero pulse source tunable in wavelength with a single- or multiwavelength output based on four-wave mixing in a newly developed highly nonlinear fiber," Electron. Lett., vol. 13, pp. 70-72, 2001.

[75] P. A. Andrekson, N. A. Olsson, J. R. Simpson, T. Tanbun-Ek, R. A. Logan, and M. Haner, "16 Gbit/s all-optical demultiplexing using four-wave mixing," Electron. Lett., vol. 27, pp. 922-924, 1991.

[76] H. Sunnerud, M. Westlund, J. Li, J. Hansryd, M. Karlsson, P.-O. Hedekvist, and P. A. Andrekson, "Long-term $160 \mathrm{~Gb} / \mathrm{s}-\mathrm{TDM}$, RZ transmission with automatic PMD compensation and system monitoring using an optical sampling system," in Proc. Eur. Conf. Optical Communication, Amsterdam, The Netherlands, 2001, Postdeadline Paper, M.1.9.

[77] H. Ohta, S. Nogiwa, Y. Kawaguchi, and Y. Endo, "Measurement of 200 Gbit/s optical eye diagram by optical sampling with gain switched optical pulse," Electron. Lett., vol. 36, pp. 737-739, 2000.

[78] H. Takara, S. Kawanishi, A. Yokoo, S. Tomaru, T. Kitoh, and M. Saruwatari, "100 Gbit/s optical signal eye-diagram measurement with optical sampling using organic nonlinear optical crystal," Electron. Lett., vol. 32, pp. 2256-2258, 1996.

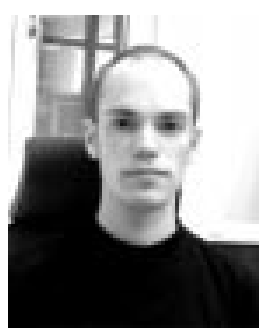

systems.

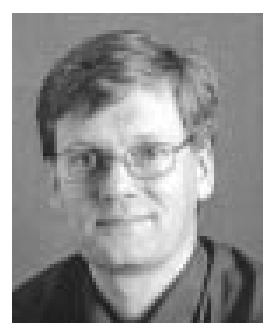

Peter A. Andrekson (S'84-M'88) was born in Göteborg, Sweden, in 1960. He received the Ph.D. degree from Chalmers University of Technology, Sweden, in 1988

He spent one year in the organizing committee for the European Conference on Optical Communication (ECOC) held in Göteborg in 1989. After about three years with AT\&T Bell Laboratories, Murray Hill, NJ, during 1989-1992, he returned to Chalmers where he was later promoted to Associate professor. Since 2001 , he is a full professor at the Department of Microelectronics. Since November 2000, he is also the Director of Research at CENiX Inc., Allentown, PA. His research interests include nearly all aspects of high-speed and high-capacity fiber communications, such as fiber based optical amplifiers, nonlinear pulse propagation, all-optical functionalities, polarization-mode disperision, soliton transmission, etc. He is the author and co-author of over 200 scientific publications and conference papers in the area of optical communications, 40 of which were invited papers at international conferences. He served as an expert for the evaluation of the 1996 Nobel Prize in Physics. In 1993, he was awarded a substantial price from the Swedish government research committee for outstanding work performed by young scientists, and he was awarded the Telenor Nordic research award for his contribution to "optical technologies," in 2000.

Dr. Andrekson is a member of the Optical Society of America (OSA) and the Swedish Optical Society. He is currently serving on the program committees for ECOC, UEO, CLEO/USA, IOOC, and NLGW.

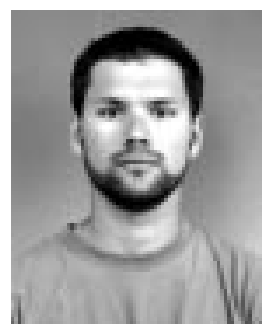

Mathias Westlund was born in Göteborg, Sweden, on March 25, 1975. He received the M. S. degree in physics in 1999, and is working toward the Ph.D. degree in electrical engineering at Chalmers University of Technology, Göteborg, Sweden.

His research is focused on high-speed optical transmission systems and applications based on nonlinear fiber effects.

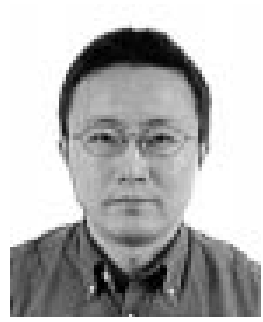

Jie Li was born in Nanjing, China, on October 21, 1966. He received the B.S. degree in physics from Nanjing University, Nanjing, China, the M.S. degree in electrical engineering from Chalmers University of Technology, Göteborg, Sweden, and the Licentiate of Engineering degree 1989, 1998 and 2001, respectively. He is presently working toward the Ph.D. degree.

Currently, he is with Ericsson's Optical Network Research Laboratory, Stockholm, Sweden. His research interests include optical-fiber based ultrashort optical pulse generation and transmission, all-optical sampling, and demultiplexing.

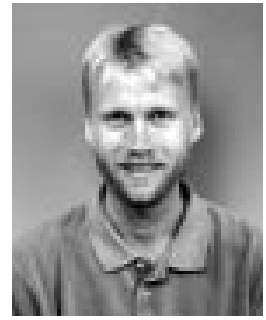

Per-Olof Hedekvist was born in Mölndal, Sweden, on Oct. 25, 1967. He recieved his M.Sc. and Ph.D. degrees from School of Electrical Engineering at Chalmers University of Technology, Göteborg, Sweden, in 1993 and 1998, respectively.

The thesis topic was four-wave mixing in optical fiber. He held a post-doc position at California Institute of Technology, Pasadena, CA, between 1998 and 2000, working on applications utilizing nonlinear effects in semiconductor optical amplifiers. He is presently assistant professor at the Department of Microelectronics, Chalmers, Sweden, where he is active in experimental research on fiber-optic transmission. 